\title{
Fermion Masses and the Standard Model Group as a Diagonal Subgroup
}

\author{
C.D. Froggatt ${ }^{a, 1}$, G. Lowe ${ }^{a}$ and H.B. Nielsen ${ }^{b}$ \\ ${ }^{a}$ Dept. of Physics and Astronomy, University of Glasgow, Glasgow' G12 8QQ, U.K. \\ b The Niels Bohr Institute, Blegdamsvej 17-21, DK 2100 Copenhagen, Denmark
}

\begin{abstract}
We argue that if the Standard Model is viewed as the effective low energy remnant of some extended group, and if the observed fermion masses are to be naturally produced as this larger group is spontaneously broken, then the extended group should possess certain characteristics. Specifically, its irreducible fermion representations should be no bigger than those of the Standard Model Group, the Standard Model Group should be embedded within it as a diagonal subgroup and its non-abelian part should be gauged. We analyse how well some groups of this type can satisfactorily account for the fermion mass and mixing hierarchies, at least order of magnitude wise.
\end{abstract}

PQDQ20684

${ }^{1}$ Research partially funded by the UK Science and Engineering Research Council 


\section{Introduction}

Despite the remarkable success of the Standard Model [1] (SM) in describing known physics, it nevertheless possesses several unsatisfactory features which demand explanation and perhaps paramount amongst these is the profusion of free parameters related to fermion masses and mixing angles. The masses themselves are merely accommodated in the SM via 9 Yukawa couplings while 4 parameters of the CKM matrix describe the mixing of quark gauge eigenstates to form mass eigenstates; none of these parameters is predicted in the SM and a strong hierarchical structure is observed in Nature. For example, if we take the fermion masses to be:

$$
m_{i}=y_{i}\langle\phi\rangle_{w s}
$$

where the Weinberg-Salam Higgs VEV is $\langle\phi\rangle_{\mathrm{ws}}=174 \mathrm{GeV}$, then the Yukawa couplings $y_{i}$ have orders of magnitude:

$$
\begin{aligned}
y_{t} \sim 10^{0}, y_{c} & \sim y_{b} \sim y_{\tau} \sim 10^{-2}, y_{s} \sim y_{\mu} \sim 10^{-3}, \\
y_{d} \sim y_{u} & \sim 10^{-4}, y_{\epsilon} \sim 10^{-5}
\end{aligned}
$$

It is particularly distasteful to find these arbitrary Yukawa couplings ranging over 5 orders of magnitude; all fermion masses are naively expected to be of order $\langle\phi\rangle_{\text {ws }}$ but patently most are much lighter than this. This suppression of the masses relative to their natural scale surely constitutes our most promising window on physics at higher energy scales - we take the view that the Standard Model Group $(S M G \equiv S U(3) \times S U(2) \times U(1))$ is only a low energy remnant of some larger group $G$ and the fermion mass hierarchy a consequence of the spontaneous breaking of $G$ to the SMG.

The number of SM extensions studied in the literature is, of course, very large. Probably most popular have been Grand Unified Theories [2-4] (GUTs) of various types in which quarks and leptons are granted equal status, different fermion irreducible representations (IRs) of the SMG being grouped in single IRs of the (usually simple) group $G$. Nowadays, 
supersymmetric GUTs $[5,6]$ are the focus of much attention due to their fitting of accurate LEP data for $\alpha_{s}, \alpha$ and $\sin ^{2} \theta_{W}$. Unfortunately the general GUT treatment of fermion masses can only be described as unsatisfactory: typically, the mass hierarchy is merely incorporated by adopting some mass matrix ansatz usually of a generalised Fritzsch structure [7] (see e.g. [8]) which already has the hierarchy built in via hugely differing Yukawa couplings. This cannot be truly said to "solve" the fermion mass problem. Despite some attempts to properly incorporate the generations within a GUT scenario (e.g. [9]), this "generation problem" has usually been addressed in models featuring a different approach to SMG extensions, that of "horizontal symmetry" [10-12] which relates corresponding members of the different generations, and these models have tried to shed some light on the fermion mass and mixing hierarchies with limited success (see e.g. [13]). In this paper we seek a natural order of magnitude understanding of these hierarchies (i.e. one which does not depend on an arbitrary choice of vastly differing Yukawa couplings) based on a type of SMG extension very different from these more popular candidates.

A brief presentation of some of our models was given in [14], but here we will give a full discussion of both the motivation underlying our choice of SMG extension and the analysis of our models. After a statement of our main assumptions in Section 2, we classify the SMG extensions (Section 3) according to how they treat the IRs of the SMG, finding that the type of models which intuitively seem to offer the greatest promise of explaining the salient features of the fermion mass hierarchy are in fact least popular in the literature. This is the class of extensions characterised by having the IRs of the extended group $G$ coincident with those of the SMG itself. Section 4 then covers the embedding of the SMG in $G$ (or some subgroup of $G$ ) as a diagonal subgroup and the consequent gauging of $G$. A general discussion of these models is provided in Section 5 leading to arguments for specific cases in Section 6. Finally, after some some discussion of numerology (Section 7) and method (Section 8), our results for these cases are given in Section 9. 


\section{Assumptions \& Rationale for Mass Suppression}

The first of our basic assumptions is that there exist no mass-protected fermions which provide a net non-zero contribution to gauged or mixed anomalies, other than those of the 3 generation SM. A fermion is said to be "mass-protected" by a symmetry group $G$ if its left- and right-handed components lie in inequivalent representations of $G$; if $G$ is exactly conserved then the Lagrangian is forbidden to contain a mass term for such a fermion. In the case of "light" fermions i.e. those mass-protected by the SMG itself, this assumption has a good chance of being true due to the familiar LEP result that there are only 3 light neutrinos and the fact that if "new" fermions with mass of order $\langle\phi\rangle_{\text {ws }}$ existed we might hope to have seen at least indirect signs of it. But even for fermions which are vector-like under the SMG and are only mass-protected by a symmetry $G$ broken at some high scale $M \gg\langle\phi\rangle_{\text {ws }}$ (so that the fermions would naturally have a mass of order $M$ ), this is still a desirable assumption. If we drop it and allow more fermions then the number of models we could postulate is limitless, with no experimental evidence to aid our selection - we do not wish to become embroiled in such a process.

We also assume throughout our analysis that the 3rd generation symmetry eigenstates $(t, b, \tau)$ are approximately equal to the respective 3 rd generation mass eigenstates i.e. if the fermion mass terms generated in the Yukawa sector of the Langrangian are:

$$
\mathcal{L}_{M}=\overline{U_{R}} M_{U} U_{L}+\overline{D_{R}} M_{D} D_{L}+\overline{l_{R}} M_{l} l_{L}+\text { h.c. }
$$

and $U=(u, c, t), D=(d, s, b)$ and $l=(e, \mu, \tau)$ then the 3rd generation masses receive their dominant contribution from the respective $M_{i}(3,3)$. This natural assumption is a feature of virtually all models of fermion mass matrices (e.g. the Fritzsch matrices [7]) and, despite the fact that we explicitly dropped it in a recent article [15], we wish to maintain it here as by far the simplest approach to fermion mass matrices. It also allows us to make statements about the 3rd generation masses purely from knowledge of the symmetry transformation properties of the corresponding Weyl states. Henceforth we will 
use the term "generation" to decribe both the symmetry and mass eigenstates; it will always be clear from the context exactly which type of states we are referring to.

Finally, we demand that all order of magnitude features of the observed fermion mass hierarchy should be potentially explainable from the dynamics of any particular model. In particular, they must not be results of a hierarchy in fundamental Yukawa couplings; indeed, we shall take all such couplings to be $O(1)$ (we return to this point later) so that they are responsible only for fine structure. What we have in mind is a situation where some subgroup of the full chiral symmetry group $G$ is "partially conserved" i.e. only weakly broken (the component symmetries of the relevant subgroup are "partially conserved chiral symmetries" or PCCSs). The left- and right-handed fermion components which couple to form mass matrix elements will, in general, have different transformation properties under one or more PCCSs and these elements will consequently be suppressed. Then, even though all fundamental Yukawa couplings in the "true" underlying theory may be $O(1)$, the effective Yukawa couplings of the fermions to the Weinberg-Salam Higgs in the familiar low energy theory can naturally be small. This is precisely the scenario envisaged in [16] and [17] where the $S M G$ is augmented by an abelian "flavour symmetry" $U(1)_{f}$ which is taken to be partially conserved (similar extensions of $S O(10)$ GUT models have also been considered [18]). Note that although abelian symmetries different to the weak hypercharge of the SMG have often been called horizontal symmetries in the literature, we avoid this terminology, reserving the term "horizontal" to describe only non-abelian symmetries for reasons which will become clear in the next section.

It is convenient here to note that we do not assume from the outset that the full symmetry group $G$ is gauged. In Section 4 it will emerge that the type of $G$ argued for in Section 3 as being most favourable for the generation of the fermion mass hierarchy (or at least the non-abelian part of such a group) can in fact be proven to be a local symmetry group. 


\section{SMG Extensions}

We seek the extensions most naturally suggested by the known fermion masses and quark mixing angles, and we assume that only the 45 Weyl fermions of the SM exist in the low energy regime. Furthermore, we are only interested in that part of any extension which acts non-trivially on at least one of these 45 states (we now take $G$ to be this non-trivial group) i.e. letting $\hat{G}$ denote the subgroup of the true fundamental gauge group which transforms these 45 states among themselves, we are only interested in:

$$
G=\hat{G} / L
$$

where $L$ is the invariant subgroup of $\hat{G}$ consisting of those elements which act trivially on the known 45 Weyl fields. The elements of $G$ can thus be distinguished by their action on the 45 states, and so $G$ is naturally identified with a subgroup of $U(45)$, the unitary group in whose fundamental representation would sit all the known Weyl fermions. That is:

$$
S M G \subseteq G \subseteq U(45)
$$

and there essentially exists only a finite number of algebras corresponding to such $G$.

However, this will be a very large number and in the present article we suggest only a fairly crude classification of the many $G$ obeying (3.2). Clearly, the 45 Weyl states fall into IRs of $G$ each of which must include a number of the IRs of the SMG. It is thus possible to classify the extensions $G$ according to the manner in which the IRs of the SMG fit into the IRs of $G$. (Note that if $G$ is fully gauged, then many groups obeying (3.2) would lead to gauge or mixed anomalies, or to the global anomaly, and so would not be acceptable as genuine models). We introduce our classification of groups $G$ by first classifying collections of IRs of the SMG, which has 5 different IRs of type $(u d)_{L}, u_{R}, d_{R},\left(\nu_{e} e\right)_{L}$ and $e_{R}$ each occuring 3 times, once for each generation (we reserve the right to flip freely between $u_{R}$ and $u_{L}^{c}$ etc). The different types of collection are: 
(i) A collection consisting only of isomorphic IRs of the SMG (this might be called a "horizontal" collection) e.g. $\left\{u_{R}, c_{R}, t_{R}\right\}$ or $\left\{(u d)_{L},(t b)_{L}\right\}$.

(ii) A collection consisting only of non-isomorphic IRs of the SMG (this might be called a "vertical" or "grand unified" collection) e.g. $\left\{d_{L}^{c},\left(\nu_{e} e\right)_{L}\right\}$ or $\left\{(u d)_{L}, u_{L}^{c}, e_{L}^{c}\right\}$.

(iii) A collection consisting of both isomorphic and non-isomorphic IRs of the SMG (this might be called a "mixed" collection) e.g. $\left\{d_{L}^{c},\left(\nu_{e} e\right)_{L}, s_{L}^{c},\left(\nu_{\mu} \mu\right)_{L}\right\}$.

We may now classify the groups $G$ according to whether its IRs number among them collections of these 3 types (see Table 1) where, in our notation, a subscript or subscripts on a group component indicate which generations are transformed non-trivially by an element of that group component. For example, $S U_{1}(5)$ acts non-trivially (in the usual manner) only on the 1st generation while $S U_{23}(3)$ acts non-trivially (in the usual manner) only on the 2 nd and 3rd generations. So a group like $S U_{1}(5) \times S U_{2}(5) \times S U_{3}(5)$ of category $(6)$ has all the fermions grouped into the usual $\overline{5}$ and $10 S U(5)$ representations but of different $S U(5)$ components e.g. $\left(d_{L}^{c} e_{L}-\nu_{e L}\right)$ would transform like $(\overline{5}, 1,1)$ whereas $\left(s_{L}^{c} \mu_{L}-\nu_{\mu L}\right)$ would transform like $(1, \overline{5}, 1)$ etc. A more complicated example is $S U_{1}(5) \times S M G_{23} \times$ $S U_{23}(2)_{H}$ which appears in category $(2)$ and has: the 1st generation states grouped into the usual $S U(5)$ IRs $\overline{5}$ and 10 and transformed only by the $S U_{1}(5)$ component; and the 2nd and 3rd generation states behaving exactly as in the SM under $S M G_{23}$ but grouped horizontally under $S U_{23}(2)_{H}$ in one of the scenarios catalogued in [13].

This table displays all combinations of the 3 different types of collection and gives one or two examples in each case. These include the usual GUT models which have no "extra" fermions (really only $S U(5)$ but this can be distorted e.g. to $S U_{1}(5) \times S U_{2}(5) \times S U_{3}(5)$ ) in category (6), the horizontal symmetry models (mentioned in the introduction) in category (4) and combinations of these two in category (7).

The extensions we wish to focus on, however, lie in category (8). Several of the nontrivial extensions in this category display most clearly a feature which is prominent throughout the table, that different subgroups of the extension transform different gener- 


\begin{tabular}{|c|c|c|c|c|}
\hline \multirow{4}{*}{$\begin{array}{c}\text { Horizontal } \\
\text { Collection(s) }\end{array}$} & \multirow{2}{*}{$\begin{array}{c}\text { Vertical } \\
\text { Collection(s) }\end{array}$} & $\begin{array}{c}\text { Mixed } \\
\text { Collection(s) }\end{array}$ & $(1)$ & $\begin{array}{c}\text { Need } \geq 5 \text { generations } \\
\text { for anomaly-free model }\end{array}$ \\
\hline & & $\begin{array}{l}\text { No Mixed } \\
\text { Collections }\end{array}$ & $(2)$ & $S U_{1}(5) \times\left\{S M G \times S U(2)_{H}\right\}_{23}$ \\
\hline & \multirow{2}{*}{$\begin{array}{l}\text { No Vertical } \\
\text { Collections }\end{array}$} & $\begin{array}{c}\text { Mixed } \\
\text { Collection(s) }\end{array}$ & $(3)$ & $\begin{array}{l}\text { Need } \geq 4 \text { generations } \\
\text { for anomaly-free model }\end{array}$ \\
\hline & & $\begin{array}{l}\text { No Mixed } \\
\text { Collections }\end{array}$ & $(4)$ & $\begin{array}{c}S M G \times S U(2)_{H} \\
U(2) \times S U(2)_{H} \times S U_{1}(3) \times S U_{23}(3)\end{array}$ \\
\hline \multirow{4}{*}{$\begin{array}{r}\text { No Horizontal } \\
\text { Collection }(\mathrm{s})\end{array}$} & \multirow{2}{*}{$\begin{array}{c}\text { Vertical } \\
\text { Collection(s) }\end{array}$} & $\begin{array}{c}\text { Mixed } \\
\text { Collection(s) }\end{array}$ & $(5)$ & $S U_{1}(5) \times\left\{S U(5) \times S U(2)_{H}\right\}_{23}$ \\
\hline & & $\begin{array}{l}\text { No Mixed } \\
\text { Collections }\end{array}$ & $(6)$ & $\begin{array}{c}S U(5) \\
S U_{1}(5) \times S U_{2}(5) \times S U_{3}(5)\end{array}$ \\
\hline & \multirow{2}{*}{$\begin{array}{l}\text { No Vertical } \\
\text { Collections }\end{array}$} & $\begin{array}{c}\text { Mixed } \\
\text { Collection(s) } \\
\end{array}$ & $(i)$ & $\begin{array}{c}S U(5) \times S U(2)_{H} \\
S M G_{1} \times\left\{S U(5) \times S U(2)_{H}\right\}_{23}\end{array}$ \\
\hline & & $\begin{array}{l}\text { No Mixed } \\
\text { Collections }\end{array}$ & $(8)$ & $\begin{array}{c}S M G \\
S M G_{1} \times S M G_{2} \times S M G_{3}\end{array}$ \\
\hline
\end{tabular}

Table 1

Classification of SMG extensions according to the manner in IRs of the SMG fit into IRs of $G$. Subscript "H" means "horizontal" while subscript "ij" means that the relevant group component acts only on generations " $\mathrm{i}$ " and " $\mathrm{j}$ ". Some examples of each category are given, and these are gauge and mixed anomaly free so that they may be considered valid gauge groups. Note that abelian flavour symmetries may be added on to any group. although the corresponding charges are constrained if anomalies have to be cancelled.

ations with the corresponding part of the SMG as the "diagonal" subgroup: the subgroup whose elements correspond to identical transformations on each generation. (The purest such extension, the "anti-grand unified" gauge group $S M G^{3}$ seen here in category (8), has been considered previously $[19,20]$ and its possible relevance to the fermion mass problem suggested [21] and analysed [15]). The diagonal subgroup of a cross product 
$H^{3} \equiv H \times H \times H$ is easily seen to be:

$$
H_{\text {diag }}=\{(h, h, h): h \in H\}
$$

but even for cases where the cross product factors are not themselves isomorphic, but contain only subgroups which are isomorphic, it is possible to construct a generalised diagonal subgroup. For example, category (6) contains the group $S U_{1}(5) \times S M G_{23}$; the SMG is embedded in this as a generalised diagonal subgroup in the sense that it appears as:

$$
S M G=\{(T(h), h): h \in S M G\}
$$

where $T(h)$ is the element of $S U(5)$ corresponding to the element $h$ of the SMG when the SMG is embedded in $S U(5)$ in the standard Georgi-Glashow manner. The defining characteristic of the groups in category (8), of course, is that their IRs are coincident with those of the SMG, but the prominence of the diagonal subgroup idea throughout the table renders slightly curious the fact that this type of extension is the least popular in the literature. Obviously, the goal of GUTs to obtain a simple group $G$ completely precludes consideration of these models, but even attempts to extend the SMG in an effort to "explain" the fermion mass hierarchy have largely ignored them too, concentrating instead on the horizontal models of category (4).

This relative lack of interest is even more surprising since we may intuitively argue that Nature in some sense favours category (8) models. When examining the SM in an attempt to glean clues regarding its extension, the most remarkable feature is arguably not the near-coincidence of the running gauge couplings at some high energy scale [6] but is the presence of three generations of fermions with large mass gaps between them. The two outstanding features of this hierarchy are:

- all fermions except the top quark are light compared to the electroweak scale $\langle\phi\rangle_{\mathrm{ws}}=$ $174 \mathrm{GeV}$;

- the average mass of each generation gets successively larger, by a factor of order 60 . 
No pair of corresponding particles from different generations is degenerate, even order of magnitude wise, and consequently it is reasonable to expect that Nature has chosen a model in which the chance of such degeneracy is very low.

This argument would seem to disfavour the use of non-abelian horizontal flavour groups to account for the generation gaps: for example, the positioning of corresponding pairs of states such as $\left\{\epsilon_{R}, \mu_{R}\right\}$ into doublets of $S U(2)_{H}$ (in the $S M G \times S U(2)_{H}$ model), or even $\left\{u_{R}, c_{R}, t_{R}\right\}$ into triplets of $S U(2)_{H}$, creates the obvious risk of these states forming degenerate particles. It is however possible to make a clever choice of Higgs representations and let some subgroup of $S U(2)_{H}$ be better conserved than the rest - see [13] for example but it is hardly a natural feature of these models. To avoid inviting these degeneracies the extended group $G$ should thus have no IRs which constitute horizontal or mixed collections of IRs of the SMG.

Really, in order for $G$ to account for the large generation gaps, there must be some difference in the quantum numbers of particles in different generations (unlike the standard $S U(5)$ GUT, for example, where the generations are simple copies of each other as far as their symmetry properties are concerned) but without grouping IRs of the SMG horizontally i.e. corresponding particles from different generations should belong to inequivalent IRs of $G$. The crudest and most obvious way of achieving this is to extend the $S M G$ to $S M G \times U(1)_{f}$ and arrange the new charges $Q_{f}$ to vary from generation to generation (such an $S M G \times U(1)_{f}$ model was considered in [10], [16] and [17]). For example, a set of charges satisfying:

$$
\left|Q_{f}\left(u_{L}\right)-Q_{f}\left(u_{R}\right)\right|>\left|Q_{f}\left(c_{L}\right)-Q_{f}\left(c_{R}\right)\right|>\left|Q_{f}\left(t_{L}\right)-Q_{f}\left(t_{R}\right)\right|
$$

might naturally account for:

$$
m_{u} \ll m_{c} \ll m_{t}
$$

if the $U(1)_{f}$ was partially conserved. A more subtle method of achieving the same goal would be to extend the $\mathrm{SU}(2)$ sector of the $S M G$ to $S U_{1}(2) \times S U_{2}(2) \times S U_{3}(2)$ so that $(u d)_{L}$ transforms as $(2,1,1),(c s)_{L}$ as $(1,2,1)$ and $(t b)_{L}$ as $(1,1,2)$ while $u_{R}, c_{R}$ and $t_{R}$ 
would transform as $(1,1,1)$. Then taking $S U_{i}(2)$ to be better conserved with decreasing $i$ so that the matrix element connecting $u_{L}$ and $u_{R}$ is more strongly suppressed than that connecting $c_{L}$ and $c_{R}$ etc again might naturally produce (3.6).

Furthermore, if we consider the quark mass gaps within generations as "big" then we should beware of collecting more than single IRs of the SMG to form IRs of $G$ i.e. we should be very cautious about using vertical collections. In the usual $S U(5)$ model, the suppression of $m_{b}$ w.r.t. $m_{t}$ and of $m_{s}$ w.r.t. $m_{c}$ is obtained via the Yukawa couplings, just as in the SM. But there are other category (6) possibilities $\left(e . g . S U(5) \times U(1)_{f}\right.$, $\left.S U_{1}(5) \times S U_{23}(5) \times U(1)_{f}\right)$ which have to be considered, although these do not seem promising $[17,22]$. In this paper, we therefore suggest that only category (8) models are "favoured" by Nature, at least as far as generating the fermion mass hierarchy is concerned.

\section{Embedding the SMG and Gauging G}

In this section we look more closely at the groups of category (8), demonstrating that the $S M G$ is embedded within each of them as a diagonal subgroup (despite a threat posed by the left-handed quarks) and arguing that their non-abelian parts should consequently be gauged.

Consider, then, one IR $\Psi$ of a category (8) group, whose IRs are no bigger than those already present in the SM. Leaving aside for the moment the left-handed quarks, we find that all possible unitary transformations of the Weyl components forming $\Psi$ are in fact "already" performed by some SMG gauge transformation ${ }^{1}$. For example, a righthanded quark with electric charge $+2 / 3\left(\right.$ e.g. $\left.\Psi=u_{R}\right)$ is a 3 -dimensional IR and the

${ }^{1}$ It has been argued [23] that in order to understand the existence of 4 space-time dimensions as a result of the specially high stability of the Weyl equation in $3+1 \mathrm{~d}$, it is necessary that this is true viz. that all possible transformations are realised by some gauge degrees of freedom. 
set of all unitary transformations of $\Psi$ consequently forms a $U(3)$ group. The $S U(3)$ subgroup of these transformations can as far as the transformation of $\Psi$ is concerned be identified with the familiar colour $S U(3)$ transformations, and the $U(1)$ subgroup (the overall phase) can similarly be identified with the weak hypercharge phase rotation. If we next look at $\Psi^{\prime}=c_{R}$ (say), we can obviously make the same claim but $\Psi$ and $\Psi^{\prime}$ could have their $S U(3)$ (or $U(1)$ ) transformations performed independently so that the overall transformations of $\Psi+\Psi^{\prime}$ would form the group $S U^{u_{R}}(3) \times S U^{C_{R}}(3)$ (in this suggestive notation the superscript indexes which representation is non-trivially transformed by the group component in question). So, taking account of all $\Psi$ except the left-handed quarks, the transformations forming a category $(8)$ group can be described by some subgroup of a group $G$ which looks like:

$$
G=\prod_{i} S U^{i}(3) \times \prod_{j} S U^{j}(2) \times \prod_{k} U^{k}(1)
$$

where $i$ runs over right-handed quarks, $j$ over left-handed leptons and $k$ over right-handed quarks and all leptons. Each component acts just like its SM counterpart, but on one IR only.

Note that this group is considerably more fragmented than the examples given in Table 1 (e.g. $\left.S M G_{1} \times S M G_{2} \times S M G_{3}\right)$ where each generation had its own group component here, each irreducible representation has its own group component.

The case of $\Psi$ being a 6-dimensional IR of left-handed quarks, however, suggests the possibility of unitary transformations (viz. $U(6)$ or $S U(6)$ ) which are not identifiable with SM gauge transformations (restricted to $\Psi$ ). For example, for the IR $(u d)_{L}$ the SM gauge transformations can: change colour of the $u$ and $d$ quarks simultaneously $\left(e . g . u_{L}^{r} \leftrightarrow u_{L}^{b}\right.$ at the same time as $\left.d_{L}^{r} \leftrightarrow d_{L}^{b}\right)$; the weak isospin of all colours simultaneously $\left(e . g . u_{L}^{r} \leftrightarrow d_{L}^{r}\right.$ $\left.u_{L}^{b} \leftrightarrow d_{L}^{b}, u_{L}^{g} \leftrightarrow d_{L}^{g}\right)$; or some combination of these (e.g. $\left.u_{L}^{r} \leftrightarrow d_{L}^{b}, u_{L}^{b} \leftrightarrow d_{L}^{r}, u_{L}^{g} \leftrightarrow d_{L}^{g}\right)$. But they cannot change the weak isospin of some colours while leaving others untouched (e.g. $u_{L}^{r} \leftrightarrow d_{L}^{r} u_{L}^{b} \leftrightarrow d_{L}^{b}, u_{L}^{g} \leftrightarrow u_{L}^{g}, d_{L}^{g} \leftrightarrow d_{L}^{g}$ ) which an $S U(6)$ transformation would 
in general be able to do. As far as our category (8) group is concerned, though, all we

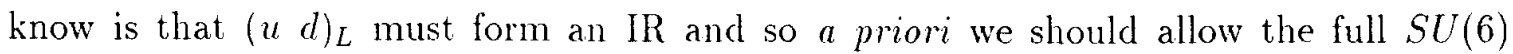
transformations. But taking a set of transformations forming a global SU(6) symmetry and commuting members of this set with SM gauge transformations yields a gauged $\mathrm{SU}(6)$ symmetry acting on $\Psi$, a scenario forbidden by the requirement that a viable model should have no net gauge anomalies. The $S U(3) \times S U(2)$ part of the $S M G$ has to be embedded in $S U(6)$ in a manner independent of which IR we consider, and so if we have one lefthanded quark representation $\Psi$ transforming as a 6 under $S U(6)$ it is not possible to have another $\Psi^{\prime}$ transforming as a $\overline{6}$ in an effort to cancel anomalies. It follows that we cannot include an $S U(6)$ component in an extended anomaly-free gauge group $G$; all we can really do in category (8) groups for left-handed quarks is to retain the usual $S U(3) \times S U(2)$ transformations but, as in (4.1), allow different "copies" for different IRs.

Overall, then, category ( 8 ) groups $G$ are essentially subgroups of:

$$
G^{\prime}=\prod_{i} S U^{i}(3) \times \prod_{j} S U^{j}(2) \times \prod_{k} U^{k}(1)
$$

where $i$ runs over all quark representations, $j$ over all left-handed representations and $k$ over all 15 representations in the SM. Again, each component acts just like the corresponding SMG component but on one IR only. We do not let $i$ and $j$ run over all 15 representations because we wish to maintain consistency with (3.1) where we stated that we want no part of our group to act trivially on all known states and this is exactly what components like $S U^{\epsilon_{R}}(3)$ and $S U^{s_{R}}(2)$ would do. Nevertheless, allowing for this technicality, the $S M G$ is identifiable for each IR with one $S U(3) \times S U(2) \times U(1)$ copy, so that the $S M G$ is realised as a subgroup of $G^{\prime}$ in which the group elements corresponding to each copy are identical. But this is nothing other than the diagonal subgroup discussed earlier and we can thus claim that the 3 factors $U(1), S U(2)$ and $S U(3)$ of the $S M G$ occur as diagonal subgroups of the corresponding parts of $G$ for all $G$ in category (8). Note that some of these factors may be embedded in $G$ as trivial diagonal subgroups e.g. for a group 
like:

$$
G=S U(3) \times S U^{1}(2) \times S U^{2}(2) \times \ldots \times S U^{6}(2) \times U(1)
$$

where the superscripts index the 6 known left-handed IRs of the SM, the $S U(2)$ part of the $S M G$ is embedded as a true diagonal subgroup while the embedding of the $S U(3)$ and $U(1)$ parts is trivial.

Note that nothing we have said actually prevents us from tagging abelian flavour symmetries onto the $G^{\prime}$ of $(4.2)$ and the concept of a diagonal subgroup is not clean for abelian groups. Which subgroup of a product $U(1)^{n}$ is termed the "diagonal" subgroup depends on which linear combination of generators is chosen to form a basis. For example, if the group $S U^{1}(2) \times S U^{2}(2)$ has generators $I_{i}^{1}$ and $I_{j}^{2}(i, j=1,2,3)$ then the diagonal subgroup $S U(2)_{\text {diag }}$ is unambiguously defined to be generated by $I_{i}^{1}+I_{i}^{2}(i=1,2,3)$. But the same cannot be said of the group $U^{1}(1) \times U^{2}(1)$ generated by $Y_{1}$ and $Y_{2}$ : if we switch basis e.g. to $Y_{1}^{\prime}$ and $Y_{2}^{\prime}$ where:

$$
\begin{aligned}
& Y_{1}^{\prime}=Y_{1}+Y_{2} \\
& Y_{2}^{\prime}=Y_{1}-Y_{2}
\end{aligned}
$$

then (following the non-abelian construction) we have two equally valid possibilities for the generator of our supposed diagonal subgroup $U(1)_{\text {diag }}$ viz. $Y_{1}+Y_{2}$ and $Y_{1}^{\prime}+Y_{2}^{\prime}$ which generate entirely different subgroups. Thus the concept is basis dependent and so use of the expression "diagonal" subgroup is a notational matter devoid of physical content.

Nevertheless, a strong consequence of concluding that the non-abelian part of the $S M G$ $\left(S M G_{\text {na }}\right)$ is embedded in the non-abelian part of $G^{\prime}\left(G_{\text {na }}^{\prime}\right)$ as a diagonal subgroup is that $G_{\mathrm{na}}^{\prime}$ must be gauged. Commuting the $S M G_{\mathrm{na}}$ gauge transformations with the (a priori global) non-SMG members of $G_{n a}^{\prime}$ generates a fully gauged $G_{n a}^{\prime}$. This line of argument for gauging is obviously not applicable to any abelian factors in $G^{\prime}$ but, as the non-abelian and SM weak hypercharge symmetries are gauged, we consider it reasonable to gauge all of the abelian factors too. As it stands in (4.2), then, the now fully gauged $G^{\prime}$ is riddled 
with anomalies. Its biggest anomaly-free subgroup is:

$$
G^{\prime \prime}=S M G_{1} \times S M G_{2} \times S M G_{3}
$$

but, as previously stated, we can still add abelian flavour symmetries. The anomaly cancellation conditions for a $U(1)_{f}$ tagged onto $G^{\prime \prime}$ were given in [15] where it was further argued that they had only one solution which was relevant to our mass suppression framework so that the largest relevant anomaly-free group in category (8) is:

$$
G^{\prime \prime \prime}=S M G^{3} \times U(1)_{f}
$$

There are in fact 6 possible solutions to the no-anomaly equations for the $U(1)_{f}$ of $(4.6)$ : (i) 3 have the fermions charged just as they are under $U_{a}(1)(a=1,2,3)$ and so have nothing new to offer as far as mass suppression is concerned. If we have a $U(1)_{f}$ generated by $Y_{f}$ which is an exact copy of $U_{3}(1)$ (say) generated by $Y_{3}$ then we can make a change of basis to:

$$
\begin{gathered}
Y=Y_{3}+Y_{f} \\
Y^{\prime}=Y_{3}-Y_{f}
\end{gathered}
$$

This leaves us with a $U(1)$ generated by $Y$ which is identical to $U_{3}(1)$ and $U(1)_{f}$, and a $U(1)$ generated by $Y^{\prime}$ which acts trivially on all Weyl states and so can be dropped as in

(ii) and 3 solutions which, after a relabelling of the Weyl states are both equivalent to:

$$
\begin{aligned}
\left(u_{L}, u_{R}, d_{R}, \epsilon_{L}, \epsilon_{R}\right) & =(0,0,0,0,0) \\
\left(c_{L}, c_{R}, s_{R}, \mu_{L}, \mu_{R}\right) & =(0,1,-1,0,-1) \\
\left(t_{L}, t_{R}, b_{R}, \tau_{L}, \tau_{R}\right) & =(0,-1,1,0,1)
\end{aligned}
$$

If we try to extend the group in (4.6) to $S M G^{3} \times U(1)_{f} \times U(1)_{f}^{\prime}$ then anomaly cancellation can occur only if the $U(1)_{f}^{\prime}$ is a copy of one of these 6 solutions which is consequently irrelevant for the reasons discussed above (in fact the $U(1)_{f}^{\prime}$ can be a linear combination of the solutions in (i), or of a solution in (ii) and those in (i), but our argument still holds). We recall that the group given in (4.6) (or one of its subgroups) is only the factor group 
given by (3.1) and may thus only be part of the full group beyond the SMG.

Note that it is not truly accurate to say that all $G$ in category (8) are subgroups of $G^{\prime \prime \prime}$; it is more precise to say that the algebras of all such $G$ are subalgebras of that of $G^{\prime \prime \prime}$. This is because the no-anomaly conditions which have to be satisfied by the $U(1)_{f}$ vary (so that different $U(1)_{f}$ charges are possible) depending on which subalgebra of $S M G^{3}$ is chosen to form part of $G$. In fact, the smaller this subalgebra, the less stringent are these conditions - this will be important later. For example, if $G_{1}=S M G \times U(1)_{f}$ and $G_{2}=S M G^{3} \times U(1)_{f}^{\prime}$ then the anomaly-free charge sets for $U(1)_{f}$ comprise those of $U(1)_{f}^{\prime}$ plus many others - the no-anomaly conditions of the former simply form a subset of those of the latter. In fact, even this statement concerning subalgebras as opposed to subgroups is not completely true. While it is certainly true that there is only one relevant abelian symmetry which can be tagged onto $S M G^{3}$ (all further abelian symmetries must be copies of those already present), this is not true for smaller category (8) groups. For example, it is perfectly possible to conceive of a group $S M G \times U(1)_{f} \times U(1)_{f}^{\prime}$ where the $U(1)_{f}^{\prime}$ is not a copy of the $U(1)_{f}$ nor of the $U(1)_{Y}$ in $S M G$. Nevertheless, we will only use one abelian flavour symmetry in each of our models. As well as being aesthetically less satisfactory, a larger abelian sector would increase the number of parameters present in our ansätze (see Section 5) making any fit to the data less convincing.

Finally, as we have already mentioned, it is certainly possible that there is only one copy of some factor (e.g. SU(3)) in G i.e. the corresponding part of the SMG is embedded in $G$ as a trivial diagonal subgroup. However, if we wish to obtain mass splitting between generations then we cannot have the completely trivial case of the SMG as a diagonal subgroup of a cross product with only one factor. One or more charges, in addition to those in the SMG proper, have to exist and be approximately conserved in order to generate such splitting. 


\section{From PCCSs to Mass Matrix Elements}

Having stated the basic premise of our analysis, that partially conserved symmetries are responsible for the observed fermion masses, we now become more specific in stating exactly how the SM mass matrix elements of (2.1) are affected by the various PCCSs of the parent category (8) groups. A general SM fermion mass matrix element can be written:

$$
M_{U, D, l}(i, j)=y_{i j}^{U, D, l}\langle\phi\rangle_{\mathrm{ws}}
$$

where $y_{i j}$ is a dimensionless effective Yukawa coupling and $\langle\phi\rangle_{w s}$ is the usual WeinbergSalam Higgs VEV. Throughout our algebra we will absorb $\langle\phi\rangle_{\mathrm{ws}}^{-1}$ into $M(i, j)$ i.e. masses are algebraically specified in units of $\langle\phi\rangle_{\mathrm{ws}}$. The crux of our approach is that we assume the complex $y_{i j}$ can be written as:

$$
y_{i j}^{U, D, l}=\gamma_{i j}^{U, D, l} a_{i j}^{U, D, l}
$$

where $\gamma_{i j}$ is a complex number whose magnitude is $O(1)$ while $a_{i j}$ is real and:

$$
a_{i j}\left\{\begin{array}{l}
=1 \text { if } y_{i j} \text { is unsuppressed } \\
\ll 1 \text { if } y_{i j} \text { is suppressed }
\end{array}\right.
$$

We take the view that the $a_{i j}$ (which will obviously be responsible for the order of magnitude features of the fermion mass hierarchy) should be directly obtainable from the PCCSs. As will shortly be seen, each $a_{i j}$ will be related to one or more symmetry breaking parameters which may be thought of $[16,17]$ as the ratio of the symmetry breaking scale to the fundamental scale of the theory. For example, in a simple $S M G \times U(1)_{f}$ model such as $[16,17,24]$ with $U(1)_{f}$ approximately conserved, the order of magnitude of a suppressed matrix element is naturally given by a power of:

$$
\epsilon \simeq \Lambda_{f} / M \ll 1
$$

where $\Lambda_{f}$ is the scale at which the $U(1)_{f}$ is spontaneously broken (e.g. by the VEV of a scalar $S$ which is an $S M G$ singlet but has non-zero $U(1)_{f}$ charge) and $M$ is the fundamental scale of the $S M G \times U(1)_{f}$ model. 
Returning to (5.2). the $O(1) \gamma_{i j}$ are unknown and dropped from our analysis. We can then only specify any $M(i, j)$ up to an unknown $O(1)$ factor, but this is consistent with our aim of accounting only for the order of magnitude features of the mass and mixing hierarchies.

\subsection{Component Symmetries of Category (8) Groups}

The smallest non-trivial category (8) group is $S M G \times U(1)_{f}$ while the largest is $S M G^{3} \times$ $U(1)_{f}$. The 4 component symmetries of a group in this category are $S U_{a}(3), S U_{a}(2), U_{a}(1)$ $(a=1,2,3)$ and $U(1)_{f}$ and we look at each in turn.

\subsection{1. $S U_{a}(3)$}

Under an $S U_{a}(3)$ all states are singlets except the quarks of the $a^{\text {th }}$ generation which are triplets. So there are 3 types of matrix element:

(i) an element linking singlets which will obviously remain unsuppressed because there is no quantum number difference between the left- and right-handed states which form it (e.g. $M_{U}(1,2)$ for $\left.S U_{3}(3)\right)$;

(ii) an element linking triplets which will be unsuppressed for the same reason (e.g. $M_{U}(3,3)$ for $\left.S U_{3}(3)\right)$;

(iii) and an element linking a triplet and a singlet. This will be suppressed if the $S U_{a}(3)$ is partially conserved $\left(e . g . M_{U^{U}}(2,3)\right.$ for $\left.S U_{3}(3)\right)$. All such elements are naturally suppressed by the same amount.

Suppose, then, that some category ( 8 ) group has a partially conserved $S U_{1}(3)$ with symmetry breaking parameter $\delta_{1}$. The mass matrices would be given by:

$$
M_{U} \simeq M_{D} \simeq\left(\begin{array}{ccc}
1 & \delta_{1} & \delta_{1} \\
\delta_{1} & 1 & 1 \\
\delta_{1} & 1 & 1
\end{array}\right) \quad M_{l} \simeq\left(\begin{array}{lll}
1 & 1 & 1 \\
1 & 1 & 1 \\
1 & 1 & 1
\end{array}\right)
$$


and obviously all eigenvalues of $M_{i}^{\dagger} M_{i}$ (i.e. masses) are of order 1 (we emphasise that the equalities in (5.5) are only approximate due to our lack of knowledge of the $\gamma_{i j}$ in (5.2)).

When there is more than one partially conserved $S U_{a}(3)$ we assume that each is broken independently so that the overall symmetry breaking parameter is the product of all the individual ones. For example, if the group has $S U_{1}(3) \times S U_{2}(3) \times S U_{3}(3)$ as PCCSs and the symmetry breaking parameter for each $S U_{a}(3)$ is $\delta_{a}$, then the mass matrices are given by:

$$
M_{U} \simeq M_{D} \simeq\left(\begin{array}{ccc}
1 & \delta_{1} \delta_{2} & \delta_{1} \delta_{3} \\
\delta_{1} \delta_{2} & 1 & \delta_{2} \delta_{3} \\
\delta_{1} \delta_{3} & \delta_{2} \delta_{3} & 1
\end{array}\right) \quad M_{l} \simeq\left(\begin{array}{lll}
1 & 1 & 1 \\
1 & 1 & 1 \\
1 & 1 & 1
\end{array}\right)
$$

A smaller set of PCCSs is obtained by setting a particular $\delta_{a}=1$ i.e. taking some $S U_{a}(3)$ to be so strongly broken as to be irrelevant for mass suppression. From (5.6) it is clear that a mass hierarchy is unobtainable without the help of other PCCSs as all masses are still of $O(1)$.

\subsection{2. $S U_{a}(2)$}

Under an $S U_{a}(2)$ all states are singlets except the left-handed quarks and leptons of the $a^{\text {th }}$ generation which form doublets in the usual manner. There are thus 2 types of matrix element:

(i) an element linking singlets which is unsuppressed as usual (e.g. $M_{U}(1,2)$ for $\left.S U_{3}(2)\right)$;

(ii) and an element linking a doublet and a singlet which will be suppressed if the $S U_{a}(2)$ is approximately conserved $\left(\epsilon . g . M_{U}(2,3)\right.$ for $S\left(U_{3}(2)\right)$. Again, all such elements are naturally suppressed to the same degree.

There are no elements linking doublets because only left-handed states are doublets and any $M(i, j)$ links a left- and a right-handed state. 
So, if some category (8) group has $S U_{1}(2) \times S U_{2}(2) \times S U_{3}(2)$ as PCCSs and the symmetry breaking parameters are $\epsilon_{1}, \epsilon_{2}$ and $\epsilon_{3}$ respectively, then the mass matrices are:

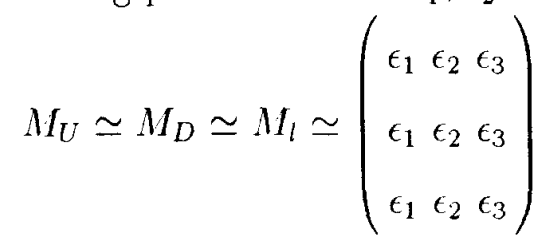

The natural order of magnitude mass predictions are:

$$
\begin{aligned}
& m_{u} \simeq m_{d} \simeq m_{e} \simeq \epsilon_{1} \\
& m_{c} \simeq m_{s} \simeq m_{\mu} \simeq \epsilon_{2} \\
& m_{t} \simeq m_{b} \simeq m_{\tau} \simeq \epsilon_{3}
\end{aligned}
$$

and so it is easy to account for the inter-generation gaps by choosing:

$$
\epsilon_{1} \ll \epsilon_{2} \ll \epsilon_{3}
$$

but mass-splitting within generations cannot be naturally obtained.

\subsection{3. $U_{a}(1)$}

To begin with, consider only one approximately conserved abelian symmetry, $U_{1}(1)$, with charges $Q_{1}$. All 2nd and 3rd generation states have $Q_{1}=0$ while the 1 st generation states have $Q_{1}$ equal to the usual weak hypercharge which we normalise to assume integer values. That is, with $Q_{1}\left(u_{R}\right) \equiv u_{R}$ etc, the $U_{1}(1)$ charges are:

$$
(u d)_{L}=1 \quad u_{R}=4 \quad d_{R}=-2 \quad\left(\nu_{\epsilon} c\right)_{L}=-3 \quad e_{R}=-6
$$

If the symmetry breaking parameter of $U_{1}(1)$ is $\lambda_{1}$ then we take the matrix elements to be given by:

$$
M(i, j) \simeq \lambda_{1}^{\left|Q_{1,}-Q_{1,}\right|}
$$

t.o obtain:

$$
M_{U} \simeq\left(\begin{array}{ccc}
\lambda_{1}^{3} & \lambda_{1}^{4} & \lambda_{1}^{4} \\
\lambda_{1} & 1 & 1 \\
\lambda_{1} & 1 & 1
\end{array}\right) \quad M_{D} \simeq\left(\begin{array}{ccc}
\lambda_{1}^{3} & \lambda_{1}^{2} & \lambda_{1}^{2} \\
\lambda_{1} & 1 & 1 \\
\lambda_{1} & 1 & 1
\end{array}\right) \quad M_{l} \simeq\left(\begin{array}{ccc}
\lambda_{1}^{3} & \lambda_{1}^{6} & \lambda_{1}^{6} \\
\lambda_{1}^{3} & 1 & 1 \\
\lambda_{1}^{3} & 1 & 1
\end{array}\right)
$$


The natural mass predictions are then:

$$
m_{u} \simeq m_{d} \simeq m_{\epsilon} \simeq \lambda_{1}^{3}
$$

while all other masses are $O(1)$.

If we extend the PCCSs to $U_{1}(1) \times U_{2}(1) \times U_{3}(1)$ then we can combine the different symmetry breaking parameters $\lambda_{1}, \lambda_{2}$ and $\lambda_{3}$ in the same way as we did for the $\delta_{a}$ in the $S U(3)^{3}$ case. That is, we can take:

$$
M(i, j) \simeq \prod_{a=1}^{3} \lambda_{a}^{\left|Q_{a i}-Q_{a j}\right|}
$$

which again means that each component symmetry is broken independently. The mass matrices would then be given by:

$$
\begin{aligned}
& M_{U} \simeq\left(\begin{array}{ccc}
\lambda_{1}^{3} & \lambda_{1}^{4} \lambda_{2} & \lambda_{1}^{4} \lambda_{3} \\
\lambda_{1} \lambda_{2}^{4} & \lambda_{2}^{3} & \lambda_{2}^{4} \lambda_{3} \\
\lambda_{1} \lambda_{3}^{4} & \lambda_{2} \lambda_{3}^{4} & \lambda_{3}^{3}
\end{array}\right) \\
& M_{D} \simeq\left(\begin{array}{ccc}
\lambda_{1}^{3} & \lambda_{1}^{2} \lambda_{2} & \lambda_{1}^{2} \lambda_{3} \\
\lambda_{1} \lambda_{2}^{2} & \lambda_{2}^{3} & \lambda_{2}^{2} \lambda_{3} \\
\lambda_{1} \lambda_{3}^{2} & \lambda_{2} \lambda_{3}^{2} & \lambda_{3}^{3}
\end{array}\right) \\
& M_{l} \simeq\left(\begin{array}{ccc}
\lambda_{1}^{3} & \lambda_{1}^{6} \lambda_{2}^{3} & \lambda_{1}^{6} \lambda_{3}^{3} \\
\lambda_{1}^{3} \lambda_{2}^{6} & \lambda_{2}^{3} & \lambda_{2}^{6} \lambda_{3}^{3} \\
\lambda_{1}^{3} \lambda_{3}^{6} & \lambda_{2}^{3} \lambda_{3}^{6} & \lambda_{3}^{3}
\end{array}\right)
\end{aligned}
$$

with order of magnitude mass predictions:

$$
\begin{aligned}
& m_{u} \simeq m_{d} \simeq m_{\epsilon} \simeq \lambda_{1}^{3} \\
& m_{c} \simeq m_{s} \simeq m_{\mu} \simeq \lambda_{2}^{3} \\
& m_{t} \simeq m_{b} \simeq m_{\tau} \simeq \lambda_{3}^{3}
\end{aligned}
$$

This is similar to the $S U(2)^{3}$ case with the generation gaps easily accounted for by taking:

$$
\lambda_{1}^{3} \ll \lambda_{2}^{3} \ll \lambda_{3}^{3}
$$

but intra-generation splitting remaining unexplained. 
The expression for $M(i, j)$ in $(5.1+)$. however. is dependent on which linear combinations of the 3 generators $Y_{1}, Y_{2}$, and $Y_{3}$ we choose to span the $U(1)^{3}$ space (it is implicitly assumed in (5.15) that the charges $Q_{a}$ correspond to the generators $\left.Y_{a}(a=1,2,3)\right)$. This is not completely satisfactory as we have no physical principle governing such a choice. What we really want instead of $(5.14)$ is an expression for $M(i, j)$ which is invariant under any basis changes. Such an expression is provided courtesy of a general metric $\mathcal{G}$ in the charge space which gives:

$$
\begin{aligned}
M(i, j) & \simeq \exp \left(-\sqrt{\left(\vec{Q}_{i}-\vec{Q}_{j}\right) \mathcal{G}\left(\vec{Q}_{i}-\vec{Q}_{j}\right)}\right) \\
& \equiv \exp \left(-\sqrt{\left(Q_{a i}-Q_{a j}\right) g_{a b}\left(Q_{b i}-Q_{b j}\right)}\right)
\end{aligned}
$$

where $\vec{Q}_{i}=\left(Q_{1 i}, Q_{2 i}, Q_{3 i}\right)$. This obviously still encompasses the motivation of (5.11) that the charge difference should be indicative of the strength of the suppression. The mass matrices generated by (5.18) are:

$$
\begin{aligned}
& M_{u} \simeq\left(\begin{array}{ccc}
\mathrm{e}^{-\sqrt{9 g_{11}}} & \mathrm{e}^{-\sqrt{16 g_{11}+g_{22}-8 g_{12}}} & \mathrm{e}^{-\sqrt{16 g_{11}+g_{33}-8 g_{13}}} \\
\mathrm{e}^{-\sqrt{g_{11}+16 g_{22}-8 g_{12}}} & \mathrm{e}^{-\sqrt{9 g_{22}}} & \mathrm{e}^{-\sqrt{16 g_{22}+g_{33}-8 g_{23}}} \\
\mathrm{e}^{-\sqrt{g_{11}+16 g_{32}-8 g_{13}}} & \mathrm{e}^{-\sqrt{g_{22}+16 g_{33}-g_{23}}} & \mathrm{e}^{-\sqrt{9_{g_{3} 3}}}
\end{array}\right) \\
& M_{d} \simeq\left(\begin{array}{ccc}
\mathrm{e}^{-\sqrt{9 g_{11}}} & \mathrm{e}^{-\sqrt{4 g_{11}+g_{22}+4 g_{12}}} & \mathrm{e}^{-\sqrt{4 g_{11}+g_{33}+4 g_{13}}} \\
\mathrm{e}^{-\sqrt{g_{11}+4 g_{22}+4 g_{12}}} & \mathrm{e}^{-\sqrt{9 g_{22}}} & \mathrm{e}^{-\sqrt{4 g_{22}+g_{33}+4 g_{23}}} \\
\mathrm{e}^{-\sqrt{g_{11}+4 g_{33}+4 g_{13}}} & \mathrm{e}^{-\sqrt{g_{22}+4 g_{33}+4 g_{23}}} & \mathrm{e}^{-\sqrt{9 g_{33}}}
\end{array}\right) \\
& M_{l} \simeq\left(\begin{array}{ccc}
\mathrm{e}^{-\sqrt{9 g_{11}}} & \mathrm{e}^{-\sqrt{36 g_{11}+9 g_{22}-36 g_{12}}} & \mathrm{e}^{-\sqrt{36 g_{11}+9 g_{33}-36 g_{13}}} \\
\mathrm{e}^{-\sqrt{9 g_{11}+36 g_{22}-36 g_{12}}} & \mathrm{e}^{-\sqrt{9 g_{22}}} & \mathrm{e}^{-\sqrt{36 g_{22}+9 g_{33}-36 g_{23}}} \\
\mathrm{e}^{-\sqrt{9 g_{11}+36 g_{32}-36 g_{13}}} & \mathrm{e}^{-\sqrt{9 g_{22}+36 g_{33}-36 g_{23}}} & \mathrm{e}^{-\sqrt{9 g_{33}}}
\end{array}\right)
\end{aligned}
$$

and should be compared with (5.15). In a large region of parameter space it is still natural to obtain expressions for the masses which are effectively the same as (5.16):

$$
\begin{aligned}
& m_{u} \simeq m_{d} \simeq m_{e} \simeq \mathrm{e}^{-\sqrt{9 g_{11}}} \\
& m_{c} \simeq m_{s} \simeq m_{\mu} \simeq \mathrm{e}^{-\sqrt{9 g_{22}}} \\
& m_{t} \simeq m_{b} \simeq m_{\tau} \simeq \mathrm{e}^{-\sqrt{9 g_{33}}}
\end{aligned}
$$


and again the generation gaps are easily explained by taking:

$$
g_{11}>g_{22}>g_{33}
$$

but splitting within generations is not accounted for. However, there are possibilities other than (5.20) obtainable from (5.19). For example, in [15] it is shown that by dropping the assumption that 3rd generation masses receive their dominant contribution from the respective $M_{i}(3,3)$ ( $\left.i=U, D, l\right)$ some kind of hierarchy within each generation can be constructed although the overall hierarchy remains unsatisfactory.

\subsection{4. $U(1)_{f}$}

The fermion charges $Q_{f}$ of the $U(1)_{f}$ must not lead to any gange or mixed anomalies, but once an anomaly-free set of charges has been found the $U(1)_{f}$ should be treated exactly as the $U_{a}(1)$ discussed previously; it is merely another ganged abelian symmetry. In particular, the charge vectors $\vec{Q}_{i}$ of $(5.18)$ are expanded in an obvious manner to $\vec{Q}_{i}=\left(Q_{1 i}, Q_{2 i}, Q_{3 i}, Q_{f i}\right)$ and the metric $\mathcal{G}$ is enlarged to encompass this extra component. Obviously no specific mass matrices can be discussed without an anomaly-free charge set so explicit examples are postponed until later.

\subsection{Putting It All Together}

We finally have to decide on some ansatz for $M(i, j)$ when several of these group components are all partially conserved. We shall in fact use 3 different ansätze. These are:

\subsubsection{Product Ansat}

Here we assume that all symmetry breaking parameters combine in product form so that:

$$
M(i, j) \simeq\left[\prod_{a=1}^{3} \delta_{a}\right]\left[\prod_{b=1}^{3} \epsilon_{b}\right]\left[\prod_{c=1}^{3} \lambda_{c}^{\left|Q_{c i}-Q_{c \jmath}\right|}\right] \lambda_{f}^{\left|Q_{f_{i}}-Q_{f \jmath}\right|}
$$


where $\lambda_{f}$ is the symmetry breaking parameter of $U(1)_{f}$. This expression is relevant for the full $S M G^{3} \times U(1)_{f}$ algebra, but we repeat that any smaller algebra can be obtained by setting particular symmetry breaking parameters equal to 1 . Note that we will always take $\left(Y_{1}, Y_{2}, Y_{3}, Y_{f}\right)$ as a basis for the $U(1)^{3} \times U(1)_{f}$ space. All models are initially analysed using this ansatz.

\subsubsection{Mired Ansatz}

Here we use a general metric in the abelian charge space to get:

$$
M(i, j) \simeq\left[\prod_{a=1}^{3} \delta_{a}\right]\left[\prod_{b=1}^{3} \epsilon_{b}\right] \exp \left(-\sqrt{\left(Q_{s i}-Q_{s j}\right) g_{s t}\left(Q_{t i}-Q_{t j}\right)}\right)
$$

where $s, t=1,2,3, f$. A smaller abelian algebra is obtained by setting particular metric elements $g_{s t}$ to 0 . All models are analysed using this ansatz.

\subsubsection{Metric Ansatz}

This will only apply to models containing PCCSs whose algebras are subalgebras of $\left[\prod_{a=1}^{3} S U_{a}(2)\right] \times\left[\prod_{b=1}^{3} U_{b}(1)\right] \times U(1)_{f}$. We would like to make a slight relaxation of the assumption that all component non-abelian symmetries are broken separately and independently of the abelian ones; after all, the $S U(2)_{L} \times U(1)_{Y}$ of the familiar electroweak theory are simultaneously broken to $U(1)_{\mathrm{em}}$ by the Weinberg-Salam Higgs field. We do this by incorporating the $S U_{a}(2)$ symmetry breaking terms into the general metric structure which previously only applied to the abelian symmetries. Specifically, we take:

$$
M(i, j) \simeq \exp \left(-\sqrt{\left(\vec{Q}_{i}-\overrightarrow{Q_{j}}\right) \mathcal{G}\left(\vec{Q}_{i}-\vec{Q}_{j}\right)}\right)
$$

where $\vec{Q}_{i}=\left(I_{1 i}^{3}, I_{2 i}^{3}, I_{3 i}^{3}, Q_{1 i}, Q_{2 i}, Q_{3 i}, Q_{f i}\right)$ and $I_{a i}^{3}$ is the usual $I^{3}$ quantum number of the $i^{\text {th }}$ state under an $S U_{a}(2)$ transformation. 


\subsection{Comments}

Note that the mixed ansatz is less suppressive than the product ansatz (the same can be said of the metric ansatz compared to the mixed one): with the natural identification

$$
\begin{aligned}
& \lambda_{c} \equiv \mathrm{e}^{-\sqrt{g_{c c}}} \quad(c=1,2,3) \\
& \lambda_{f} \equiv \mathrm{e}^{-\sqrt{g_{f f}}}
\end{aligned}
$$

any $M(i, j)$ defined by $(5.23)$ is at least as big as the corresponding element defined by (5.22). We only expect that this easing of suppression might make a real difference when in (5.22) the charge differences are large and/or two or more of the $\lambda_{i}(i=1,2,3)$ are very small. Note further that each ansatz yields the same suppression for a matrix element which is affected by only one PCCS.

Underlying all our ansätze is the assumption that there exists a complicated spectrum of superheavy fermions/scalars having $G$-invariant masses of order $M_{P}$, which can mediate all of the symmetry breaking transitions parameterised by (5.22), (5.23) and (5.24). Unlike in some $S O(10) \times U(1)$ models [18], we do not attempt to specify the superheavy spectrum. In particular, we do not assume the absence of appropriate superheavy states in order to provide texture zeros [ 7$]$ in the mass matrices.

\section{Selection of Suitable Models}

\subsection{Inter-Generation Splitting}

The first priority in the search for a model is to find one which naturally accounts for the huge mass differences between the generations - this is the single most compelling feature of the mass hierarchy. As a first approximation, we take the mass scale of each generation $\left(m_{1}, m_{2}\right.$, and $m_{3}$ respectively) to be set by its $u$-type quark; structure like 
$m_{b} \ll m_{t}$ and $m_{s} \ll m_{c}$ will be addressed later. We thus require that a set of PCCSs give:

$$
m_{1} \ll m_{2} \ll m_{3} \sim 1
$$

where we are assuming that the top mass is unsuppressed order of magnitude wise w.r.t. $\langle\phi\rangle_{\text {ws }}$. This is consistent with its expected appearance in the approximate range 100-200 $\mathrm{GeV}[25,26]$. The discussion of category (8) groups in Section 5 immediately suggests 4 candidate sets of PCCSs which might achieve this required hierarchy in the values of $m_{1}$, $m_{2}$ and $m_{3}$ :

$$
\begin{aligned}
& G_{1}^{\prime}=U_{1}(1) \times S U_{2}(2) \\
& G_{2}^{\prime}=U_{1}(1) \times U_{2}(1) \\
& G_{3}^{\prime}=S U_{1}(2) \times S U_{2}(2) \\
& G_{4}^{\prime}=S U_{1}(2) \times U_{2}(1)
\end{aligned}
$$

The full groups $G_{i}(i=1, \ldots, 4)$ corresponding to these PCCSs must then satisfy:

$$
\begin{aligned}
& H_{1} \equiv S U(3) \times S U_{2}(2) \times S U_{13}(2) \times U_{1}(1) \times U_{23}(1) \subseteq G_{1} \subseteq S M G^{3} \\
& H_{2} \equiv S U(3) \times S U(2) \times U_{1}(1) \times U_{2}(1) \times U_{3}(1) \subseteq G_{2} \subseteq S M G^{3} \\
& H_{3} \equiv S U(3) \times S U_{1}(2) \times S U_{2}(2) \times S U_{3}(2) \times U(1) \subseteq G_{3} \subseteq S M G^{3} \\
& H_{4} \equiv S U(3) \times S U_{1}(2) \times S U_{23}(2) \times U_{2}(1) \times U_{13}(1) \subseteq G_{4} \subseteq S M G^{3}
\end{aligned}
$$

in order to ensure that they contain the $S M G$ as a subgroup.

We make no mention of abelian flavour symmetries at this point. Besides their omission being aesthetically more pleasing, this is because we also have some initial motivation [19-21] for studying $S M G^{3}$ and its subgroups. Anyway, in [17] the gauge group $S M G \times$ $U(1)_{f}$ with a partially conserved $U(1)_{f}$ was dismissed as a candidate for the generation of a realistic mass hierarchy and, although the results in [24] are good for an $S M G \times$ $U(1)_{f} \times U(1)_{f}^{\prime}$ model $^{2}$ (with the abelian flavour symmetries approximately conserved), the situation regarding anomaly cancellation is unclear. It is interesting to see how far we can go in our analysis without being forced to postulate the existence of such a symmetry.

${ }^{2}$ Note that $\mathrm{H}_{2}$ has the same algebra as this group. 
The mass matrices $M_{U}^{i}, M_{D}^{i}$ and $M_{l}^{i}(i=1, \ldots 4)$ corresponding to each candidate in (6.2) are easily seen from Section 5 to be (using the product ansatz):

$$
\begin{aligned}
& M_{U}^{1} \simeq\left(\begin{array}{ccc}
\lambda_{1}^{3} & \epsilon_{2} \lambda_{1}^{4} & \lambda_{1}^{4} \\
\lambda_{1} & \epsilon_{2} & 1 \\
\lambda_{1} & \epsilon_{2} & 1
\end{array}\right) \\
& M_{D}^{1} \simeq\left(\begin{array}{ccc}
\lambda_{1}^{3} & \epsilon_{2} \lambda_{1}^{2} & \lambda_{1}^{2} \\
\lambda_{1} & \epsilon_{2} & 1 \\
\lambda_{1} & \epsilon_{2} & 1
\end{array}\right) \quad M_{l}^{1} \simeq\left(\begin{array}{ccc}
\lambda_{1}^{3} & \epsilon_{2} \lambda_{1}^{6} & \lambda_{1}^{6} \\
\lambda_{1}^{3} & \epsilon_{2} & 1 \\
\lambda_{1}^{3} & \epsilon_{2} & 1
\end{array}\right) \\
& M_{U}^{2} \simeq\left(\begin{array}{ccc}
\lambda_{1}^{3} & \lambda_{1}^{4} \lambda_{2} & \lambda_{1}^{4} \\
\lambda_{1} \lambda_{2}^{4} & \lambda_{2}^{3} & \lambda_{2}^{4} \\
\lambda_{1} & \lambda_{2} & 1
\end{array}\right) \\
& M_{U}^{3} \simeq M_{D}^{3} \simeq M_{l}^{3} \simeq\left(\begin{array}{ccc}
\epsilon_{1} & \epsilon_{2} & 1 \\
\epsilon_{1} & \epsilon_{2} & 1 \\
\epsilon_{1} & \epsilon_{2} & 1
\end{array}\right) \\
& M_{U}^{4} \simeq\left(\begin{array}{ccc}
\epsilon_{1} & \lambda_{2} & 1 \\
\epsilon_{1} \lambda_{2}^{4} & \lambda_{2}^{3} & \lambda_{2}^{4} \\
\epsilon_{1} & \lambda_{2} & 1
\end{array}\right) \quad M_{D}^{4} \simeq\left(\begin{array}{ccc}
\epsilon_{1} & \lambda_{2} & 1 \\
\epsilon_{1} \lambda_{2}^{2} & \lambda_{2}^{3} & \lambda_{2}^{2} \\
\epsilon_{1} & \lambda_{2} & 1
\end{array}\right) \quad M_{l}^{4} \simeq\left(\begin{array}{ccc}
\epsilon_{1} & \lambda_{2}^{3} & 1 \\
\epsilon_{1} \lambda_{2}^{6} & \lambda_{2}^{3} & \lambda_{2}^{6} \\
\epsilon_{1} & \lambda_{2}^{3} & 1
\end{array}\right)
\end{aligned}
$$

Natural order of magnitude mass predictions for these matrices are:

(1) $m_{3} \sim 1, m_{2} \sim \epsilon_{2}, m_{1} \sim \lambda_{1}^{3}$ or $m_{2} \sim \lambda_{1}, m_{1} \sim \epsilon_{2} \lambda_{1}^{2}$

(2) $m_{3} \sim 1, m_{2} \sim \lambda_{2}^{3}, m_{1} \sim \lambda_{1}^{3}$

(3) $m_{3} \sim 1, m_{2} \sim \epsilon_{2}, m_{1} \sim \epsilon_{1}$ and

(4) $m_{3} \sim 1$ but $m_{c, s} \sim \lambda_{2}$ while $m_{\mu} \sim \lambda_{2}^{3}$ (and $m_{1} m_{2} m_{3} \sim \epsilon_{1} \lambda_{2}^{3}$ )

respectively. The PCCSs $G_{1}^{\prime}, G_{2}^{\prime}$ and $G_{3}^{\prime}$ can thus fulfill their promise to satisfactorily provide the generation gaps whereas $C_{4}^{\prime}$ features the very poor order of magnitude prediction $m_{\mu} \simeq m_{c, s}^{3}$ and is therefore discarded at this point. Note that this prediction is unchanged if we use a different ansatz to construct the matrices because the matrix elements involved are suppressed by only one PCCS. 


\subsection{Intra-Generation Splitting}

Obviously none of the 3 remaining candidates has anything to say about mass-splitting within the generations. We are particularly interested in accounting for:

$$
\begin{aligned}
& m_{b} \ll m_{t} \\
& m_{s} \ll m_{c}
\end{aligned}
$$

as these are the most prominent order of magnitude features after (6.1). The situation concerning leptonic masses is complicated by their contrasting running behaviour as governed by the renormalisation group equations. In Section 7 we assume that our ansätze hold at some fundamental high energy scale which we take to be the Planck scale $M_{P} \simeq 10^{19} \mathrm{GeV}$ and so our order of magnitude predictions are applicable only at this scale. In [27] the fermion masses are evolved from $1 \mathrm{GeV}$ to $M_{P}$ using the SM renormalisation group equations. The order of magnitude results of this analysis of particular interest to us are:

- lepton masses change very little (they get smaller by approximately $10 \%$ )

- non-top quarks get smaller by a factor of $O(5)$.

The renormalisation group thus naturally splits the leptons from the quarks as our ansätze are evolved back down to $1 \mathrm{GeV}$. We bear this in mind throughout our algebra, concentrating for the time being on (6.5).

The only category (8) component symmetries left to us which need not suppress $M_{U}(3,3)$ (so that $m_{t}$ remains of order $\langle\phi\rangle_{w s}$ ) are $S U_{a}(3)$ and $U(1)_{f}$. A partially conserved $S U_{a}(3)$ can be immediately discarded as a possibility for generating (6.5) because, as is obvious from (5.6), it has the same effect on both $M_{U}$ and $M_{D}$. We are thus forced to conclude: the natural appearance of $a t-b$ splitting demands the introduction of an approximately conserved abelian flavour symmetry, at least if we maintain the assumption stated in Section 2 that the $3^{\text {rd }}$ generation masses receive their dominant contribution from the respective $M_{i}(3,3)(i=\mathrm{U}, \mathrm{D}, 1)$. In [15] we dropped this assumption and found that it was possible 
to obtain $m_{b} \ll m_{t}$ using the mixed ansatz, but that other sectors of the hierarchy were consequently unrealistic. Specifically, we arrived at the very poor prediction:

$$
m_{d} m_{s} m_{b} \geq m_{u} m_{c} m_{t}
$$

This argument also holds for the product ansatz, even for bases of the $U_{1}(1) \times U_{2}(1) \times$ $U_{3}(1)$ space different from $\left(Y_{1}, Y_{2}, Y_{3}\right)$. Ironically, then, we were still forced to introduce an abelian flavour symmetry in order to obtain satisfactory mass structure within all 3 generations $\left(m_{u} \simeq m_{d}, m_{s} \ll m_{c}, m_{b} \ll m_{t}\right)$. The appearance of a $U(1)_{f}$ symmetry thus seems to be unavoidable within our framework of approximately conserved chiral symmetries, with or without the assumption that $m_{t, b, \tau} \simeq M_{C, D, l}(3,3)$.

For each remaining candidate set of PCCSs (viz. $G_{1}^{\prime}, G_{2}^{\prime}$ and $G_{3}^{\prime}$ ), the no-anomaly equations for triangle diagrams involving one or more $U(1)_{f}$ gauge bosons must be solved to provide acceptable fermion charges (all other anomalies cancel because $S M G^{3}$ and its subgroups are anomaly-free in exactly the same manner as the $S M G$ itself). To do this, the groups $G_{i}(i=1,2,3)$ of $(6.3)$ must be specified, whence the full groups are $G_{i} \times U(1)_{f}$. The no-anomaly equations for $H_{i} \times U(1)_{f}$ (the $H_{i}$ are defined in $\left.(6.3)\right)$ form a subset of those for $S M G^{3} \times U(1)_{f}$. More generally, if we consider successively smaller $G_{i}$ in the chain of groups between $S M G^{3}$ and $H_{i}$ we find that each successive set of no-anomaly equations for $G_{i} \times U(1)_{f}$ forms a subset of the previous one. This is because each step down the chain involves taking the diagonal subgroup of cross product i.e. we perform one of:

$$
\begin{aligned}
S U_{a}(3) \times S U_{b}(3) & \rightarrow S U_{a b}(3) \\
S U_{a}(2) \times S U_{b}(2) & \rightarrow S U_{a b}(2) \\
U_{a}(1) \times U_{b}(1) & \rightarrow U_{a b}(1)
\end{aligned}
$$

where $a, b=1,2,3$. As far as anomaly cancellation is concerned, each such step means that we add together each corresponding pair of no-anomaly equations. For example, for the toy model $S U_{a}(3) \times S U_{b}(3) \times U(1)_{f}$ we have two anomalies to cancel: 


$$
\begin{aligned}
& \operatorname{Tr}\left[S U_{a}(3)^{2} U(1)_{f}\right]=0 \\
& \operatorname{Tr}\left[S U_{b}(3)^{2} U(1)_{f}\right]=0
\end{aligned}
$$

while for $S U_{a b}(3) \times U(1)_{f}$ we have only one:

$$
\operatorname{Tr}\left[S U_{a b}(3)^{2} U(1)_{f}\right]=\operatorname{Tr}\left[S U_{a}(3)^{2} U(1)_{f}\right]+\operatorname{Tr}\left[S U_{b}(3)^{2} U(1)_{f}\right]=0
$$

We therefore assume that $G_{i}=H_{i}(i=1,2,3)$ and once we have an anomaly-free $U(1)_{f}$ charge set for $H_{i} \times U(1)_{f}$ it is easy to check if it also constitutes an anomaly-free set for some larger group $J_{i} \times U(1)_{f}$ where $H_{i} \subset J_{i} \subseteq S M G^{3}$. So we now go on to consider the gauge groups $H_{i} \times U(1)_{f}(i=1,2,3)$ with PCCSs $U_{1}(1) \times S U_{2}(2) \times U(1)_{f}, U_{1}(1) \times U_{2}(1) \times$ $U(1)_{f}$ and $S U_{1}(2) \times S U_{2}(2) \times U(1)_{f}$ respectively.

\subsection{The $U(1)_{f}$ Fermion Charges}

The sole purpose of the abelian flavour symmetry is to provide structure within the generations, in particular the 2nd and 3rd generations. At this point, it may seen reasonable to drop our assumption that there exists only one partially conserved abelian symmetry and to consider a model like $H_{i} \times U(1)_{f} \times U(1)_{f}^{\prime}$ where $U(1)_{f}$ and $U(1)_{f}^{\prime}$ act only on the 2nd and 3rd generations respectively, providing the required structure within each generation. However, it is straightforward to show from the anomaly cancellation conditions that any abelian symmetry which affects only the $a^{\text {th }}$ generation must be a copy of the usual weak hypercharge (as far as that generation is concerned). That is, the appropriate fermion charges must be multiples of those given in (5.10) and the abelian symmetry is nothing other than $U_{a}(1)$, already dismissed as a candidate for explaining (6.5). We would therefore be forced to extend the influence of both $U(1)_{f}$ and $U(1)_{f}^{\prime}$ beyond a single generation. It is thus very awkward to have separate abelian symmetries neatly providing $m_{b} \ll m_{t}$ and $m_{s} \ll m_{c}$ without "interfering" with one another and/or disturbing already satisfactory mass relations (e.g. $m_{u} \simeq m_{d}$ ). So it seems simpler to 
maintain our original assumption and consider only a single abelian flavour symmetry, which will generate structure within both the 2nd and 3rd generations.

What about the 1 st generation? Since the $H_{i}$ already account for:

$m_{u, d, e} \ll$ all other masses

and the renormalisation group for:

$$
m_{e}<m_{u, d}
$$

the approximately conserved $U(1)_{f}$ is redundant as far as this generation is concerned. We thus take the charges $Q_{f}$ of all 1st generation Weyl states to be zero i.e. with $Q_{f}\left(u_{R}\right) \equiv u_{R}$ etc:

$$
u_{L}=u_{R}=d_{R}=\epsilon_{L}=\epsilon_{R}=0
$$

As already explained, the no-anomaly equations for any $H_{i} \times U(1)_{f}$ are easily obtained from the anomalies of the group $S M G^{3} \times U(1)_{f}$. Denoting these by $A_{i}(i=1, \ldots, 10)$ and using (6.12) we have:

$$
\begin{aligned}
A_{1}=\operatorname{Tr}\left[S U_{2}(3)^{2} U(1)_{f}\right]= & 2 c_{L}-c_{R}-s_{R} \\
A_{2}=\operatorname{Tr}\left[S U_{3}(3)^{2} U(1)_{f}\right]= & 2 t_{L}-t_{R}-b_{R} \\
A_{3}=\operatorname{Tr}\left[S U_{2}(2)^{2} U(1)_{f}\right]= & 3 c_{L}+\mu_{L} \\
A_{4}=\operatorname{Tr}\left[S U_{3}(2)^{2} U(1)_{f}\right]= & 3 t_{L}+\tau_{L} \\
A_{5}=\operatorname{Tr}\left[U_{2}(1)^{2} U(1)_{f}\right]= & c_{L}-8 c_{R}-2 s_{R}+3 \mu_{L}-6 \mu_{R} \\
A_{6}=\operatorname{Tr}\left[U_{3}(1)^{2} U(1)_{f}\right]= & t_{L}-8 t_{R}-2 b_{R}+3 \tau_{L}-6 \tau_{L} \\
A_{7}=\operatorname{Tr}\left[U_{2}(1) U(1)_{f}^{2}\right]= & c_{L}^{2}-2 c_{R}^{2}+s_{R}^{2}-\mu_{L}^{2}+\mu_{R}^{2} \\
A_{8}=\operatorname{Tr}\left[U_{3}(1) U(1)_{f}^{2}\right]= & t_{L}^{2}-2 t_{R}^{2}+b_{R}^{2}-\tau_{L}^{2}+\tau_{R}^{2} \\
\operatorname{Ag}=\operatorname{Tr}\left[U(1)_{f}^{3}\right]= & 6 c_{L}^{3}+6 t_{L}^{3}-3 c_{R}^{3}-3 t_{R}^{3}-3 s_{R}^{3} \\
& -3 b_{R}^{3}+2 \mu_{L}^{3}+2 \tau_{L}^{3}-\mu_{R}^{3}-\tau_{R}^{3} \\
A_{10}=\operatorname{Tr}\left[(\text { graviton })^{2} U(1)_{f}\right]= & 6 c_{L}+6 t_{L}-3 c_{R}-3 t_{R}-3 s_{R} \\
& -3 b_{R}+2 \mu_{L}+2 \tau_{L}-\mu_{R}-\tau_{R}
\end{aligned}
$$


In order to avoid notational confusion of these charges with the Weyl states, from now on we denote the states with a tilde $\epsilon \cdot g \cdot \tilde{t_{L}}$.

Note that the requirement:

$$
A_{2}=0
$$

which must hold for any group of which $S U_{3}(3)$ is a subgroup, is very troublesome. It simply gives ( since $\left.t_{L}=b_{L}\right)$ :

$$
\left|t_{L}-t_{R}\right|=\left|b_{L}-b_{R}\right|
$$

thus destroying any hope of generating $m_{b} \ll m_{t}$ so long as we assume that the 3 rd generation masses receive their dominant contribution from the appropriate $M_{i}(3,3)$. This is why we dropped this assumption in our analysis of the $S M G^{3} \times U(1)_{f}$ model [15].

Before using the above equations to find anomaly-free charge sets, we demand that the fermion charges satisfy certain conditions:

(i) As we have already said, we assume that (order of magnitude wise) $m_{t}$ is unsuppressed relative to $\langle\phi\rangle_{\mathrm{ws}}$. Therefore, the states ${\hat{t_{L}}}_{\text {and }} \tilde{t_{R}}$ are required to have identical transformation properties w.r.t. each PCCS, in particular the $U(1)_{f}$ symmetry. We thus take their $U(1)_{f}$ charges to be equal:

$$
t_{L}=t_{R}
$$

(ii) The good $S U(5)$ GUT scale prediction $m_{b}=m_{\tau}$, though no longer thought to be numerically exact in the minimal GUT model [28], remains an excellent order of magnitude relation. We achieve it here by demanding:

$$
\left|b_{L}-b_{R}\right|=\left|\tau_{L}-\tau_{R}\right|
$$

so that the matrix elements $M_{D}(3,3)$ and $M_{l}(3,3)$ receive the same degree of suppression. We now solve the anomaly equations using these restrictions.

\subsection{1. $H_{1} \times U(1)_{f}$}

Here the no-anomaly equations are: 


$$
\begin{aligned}
A_{1}+A_{2} & =0 \\
A_{3} & =0 \\
A_{4} & =0 \\
A_{5}+A_{6} & =0 \\
A_{7}+A_{8} & =0 \\
A_{9} & =0 \\
A_{10} & =0
\end{aligned}
$$

In this paper, we examine solutions where:

$$
\left(t_{L}, t_{R}, b_{R}, \tau_{L}, \tau_{R}\right)=-\left(c_{L} \cdot c_{R}, s_{R}, \mu_{L}, \mu_{R}\right)
$$

so that equations (6.18), (6.21), (6.23) and (6.24) are immediately satisfied (this method of cancelling the anomalies of an abelian flavour symmetry between 2 generations was used in a different context in the $S M G \times U(1)_{f}$ model of [10]). We further impose (6.16) and (6.17) to obtain 2 anomaly-free charge sets (it is the modulus signs of (6.17) which allow 2 solutions):

$$
\begin{aligned}
& \left(\begin{array}{lllll}
c_{L} & c_{R} & s_{R} & \mu_{L} & \mu_{R} \\
t_{L} & t_{R} & b_{R} & \tau_{L} & \tau_{R}
\end{array}\right)=\left(\begin{array}{rrrrr}
1 & 1 & 3 & -3 & -1 \\
-1 & -1 & -3 & 3 & 1
\end{array}\right) \\
& \text { and: } \\
& \left(\begin{array}{lllll}
c_{L} & c_{R} & s_{R} & \mu_{L} & \mu_{R} \\
t_{L} & t_{R} & b_{R} & \tau_{L} & \tau_{R}
\end{array}\right)=\left(\begin{array}{rrrrr}
1 & 1 & -3 & -3 & 1 \\
-1 & -1 & 3 & 3 & -1
\end{array}\right)
\end{aligned}
$$

Note that in this $H_{1} \times U(1)_{f}$ model the only symmetry which distinguishes the corresponding right-handed Weyl states in the 2nd and 3rd generations (e.g. $\tilde{s_{R}}$ and $\left.\tilde{b_{R}}\right)$ is $U(1)_{f}$ because all right-handed states, including those of the 2nd generation, are singlets under $S U_{2}(2)$. This means that the labelling of $\left(\tilde{s_{R}}, \tilde{b_{R}}\right)$ and $\left(\tilde{\mu_{R}}, \tilde{\tau_{R}}\right)$ is completely arbitrary (we have already defined $t_{R}$ via (6.16)). The two solutions of (6.26) and (6.27) are thus equivalent simply after relabelling these states. In order to maintain consistency with our assumption of Section 2 that the 3rd generation masses should be approximately given by $M_{i}(3,3)(i=\mathrm{U}, \mathrm{D}, 1)$, we label $\tilde{s_{R}}, \tilde{b_{R}}, \tilde{\mu_{R}}$ and $\tilde{\tau_{R}}$ as in (6.26) and this is therefore the only 
anomaly-free charge set we consider.

\subsection{2. $H_{2} \times U(1)_{f}$}

The no-anomaly equations for this model are:

$$
\begin{aligned}
A_{1}+A_{2} & =0 \\
A_{3}+A_{4} & =0 \\
A_{5} & =0 \\
A_{6} & =0 \\
A_{7} & =0 \\
A_{8} & =0 \\
A_{9} & =0 \\
A_{10} & =0
\end{aligned}
$$

and again we demand that the 3rd generation charges satisfy $(6.25)$ whence equations $(6.28),(6.29),(6.34)$ and $(6.35)$ are immediately satisfied. After imposing (6.16) and (6.17) we again find 2 anomaly-free charge sets:

$$
\begin{aligned}
& \left(\begin{array}{lllll}
c_{L} & c_{R} & s_{R} & \mu_{L} & \mu_{R} \\
t_{L} & t_{R} & b_{R} & \tau_{L} & \tau_{R}
\end{array}\right)=\left(\begin{array}{rrrrr}
5 & 5 & -1 & 1 & -5 \\
-5 & -5 & 1 & -1 & 5
\end{array}\right) \\
& \text { and: } \\
& \left(\begin{array}{lllll}
c_{L} & c_{R} & s_{R} & \mu_{L} & \mu_{R} \\
t_{L} & t_{R} & b_{R} & \tau_{L} & \tau_{R}
\end{array}\right)=\left(\begin{array}{rrrrr}
1 & 1 & 13 & 13 & 1 \\
-1 & -1 & -13 & -13 & -1
\end{array}\right)
\end{aligned}
$$

In this model the $U_{2}(1)$ adequately differentiates between all corresponding states of the 2nd and 3rd generations, so there is no ambiguity in the labelling.

\subsection{3. $H_{3} \times U(1)_{\mathrm{f}}$}

Here, the no-anomaly equations are exactly the same as for the $H_{1} \times U(1)_{f}$ model. However, the arbitrariness in the labelling of right-handed states is even greater for $\mathrm{H}_{3} \times$ 
$U(1)_{f}$, extending to the 1 st generation. This is because the $U(1)_{f}$ is the only symmetry which differentiates between any corresponding right-handed states $\left(e . g . \dot{d}_{R}, \dot{s}_{R}\right.$ and $\left.\tilde{b_{R}}\right)$. The labelling of the charges of $(6.26)$ which maintains consistency with the assumption that $M_{i}(3,3)(i=\mathrm{U}, \mathrm{D}, 1)$ provides the $3 \mathrm{rd}$ generation masses is:

$$
\left(\begin{array}{lllll}
u_{L} & u_{R} & d_{R} & \epsilon_{L} & \epsilon_{R} \\
c_{L} & c_{R} & s_{R} & \mu_{L} & \mu_{R} \\
t_{L} & t_{R} & b_{R} & \tau_{L} & \tau_{R}
\end{array}\right)=\left(\begin{array}{rrrrr}
0 & 0 & -3 & 0 & 0 \\
1 & 1 & 3 & -3 & -1 \\
-1 & -1 & 0 & 3 & 1
\end{array}\right)
$$

i.e. the original labelling of $\dot{d}_{R}$ and $\tilde{b}_{R}$ has to be switched in order to ensure that $m_{b} \simeq$ $M_{D}(3,3)$.

\section{Numerology}

In this section, we take some time out to briefly state the numerical values of the masses and mixing angles which we will attempt to fit with the parameters of our ansätze, and to discuss the procedure by which we carry out this fit.

At $1 \mathrm{GeV}$, we take the running masses to be [27]:

$$
\begin{aligned}
& m_{u}=5.2 \mathrm{MeV}, m_{c}=1.41 \mathrm{GeV}, \\
& m_{d}=9.2 \mathrm{MeV}, m_{s}=194 \mathrm{MeV}, \\
& m_{\epsilon}=0.5 \mathrm{MeV}, m_{\mu}=105 \mathrm{MeV}, m_{\tau}=1.78 \mathrm{GeV}
\end{aligned}
$$

and the physical masses (i.e. "pole" masses) to be:

$$
m_{b}^{\text {phys }}=4.99 \mathrm{G} \in V, 100 \mathrm{G} \in V \leq m_{t}^{\text {phys }} \leq 200 \mathrm{GeV}
$$

The physical mass is defined as:

$$
m_{q}^{\text {phys }}=m_{q}\left(m_{q}^{\text {phys }}\right)\left[1+\frac{4}{3 \pi} \alpha_{s}\left(m_{q}^{\text {phys }}\right)\right]
$$

where $m_{q}$ is the rumning mass. For the mixing angles at $1 \mathrm{GeV}$ we use the geometric mean of the ranges given by the Particle Data Group [29]: 


$$
\begin{aligned}
\left|V_{c k m}\right| & =\left(\begin{array}{ccc}
V_{u d} & V_{u s} & V_{u b} \\
V_{c d} & V_{c s} & V_{c b} \\
V_{t d} & V_{t s} & V_{t b}
\end{array}\right) \\
& =\left(\begin{array}{ccc}
0.9747-0.9759 & 0.218-0.224 & 0.002-0.007 \\
0.218-0.224 & 0.9735-0.9751 & 0.032-0.054 \\
0.003-0.018 & 0.030-0.054 & 0.9985-0.999 .5
\end{array}\right)
\end{aligned}
$$

Our ansätze are taken to hold at the Planck scale. This is a legacy of the anti-grand unified model of $[19,20]$ where the gauge group $S M G^{3}$ (a good symmetry at $M_{P}$ ) is spontaneously broken just below $M_{P}$ to the $S M / G$, its diagonal subgroup:

$$
S M G^{3} \stackrel{M_{P}}{\longrightarrow} S M G
$$

In fitting the parameters of our ansätze to experiment, we must therefore use data appropriate to the scale $M_{P}$. In [2خ] the masses and mixings are evolved from $1 \mathrm{GeV}$ to $M_{P}$ using SM renormalisation group equations, and we use their results to extract values at $M_{P}$ which we then take as input for our fit.

The fitting procedure itself consists of several steps:

(i) Firstly, algebraic expressions for the 3 mass matrices are obtained i.e. collectively denoting the parameter set of a particular model by $\xi$ we calculate all matrix elements as functions of $\xi, M_{i j}(\xi)$, according to one of the ansätze of Section 5. Note that all $M_{i j}$ are real and specified only up to factors of $O(1)$.

(ii) For each mass matrix we calculate $M^{\dagger}(\xi) M(\xi)$ and algebraically diagonalise it to leading order in small quantities. This will be illustrated in the next section where a matrix is explicitly diagonalised for one of our models. The diagonalisation process yields 3 eigenvalues from each matrix which give the quark/lepton masses as functions of $\xi$. For the quarks, the corresponding eigenvectors are calculated - in the generic case these give the modulus of the CKM matrix, $\left|V_{c k m}\right|$. This can be seen by considering the unitary matrices of eigenvectors $R_{u}$ and $R_{t}$ which satisfy: 


$$
\begin{aligned}
& R_{U}^{\dagger}\left(M_{U}^{\dagger} M_{U}\right) R_{U}=\operatorname{diag}\left(m_{u}^{2}, m_{c}^{2}, m_{t}^{2}\right) \\
& R_{D}^{\dagger}\left(M_{D}^{\dagger} M_{D}\right) R_{D}=\operatorname{diag}\left(m_{d}^{2}, m_{s}^{2}, m_{b}^{2}\right)
\end{aligned}
$$

The mixing matrix is then given by:

$$
V_{c k m}=R_{U}^{\dagger} R_{D}
$$

If the unknown complex Yukawa couplings of (5.2) have random phases then we expect the $\mathrm{CP}$ violating phase $\delta$ to satisfy:

$$
\sin \delta=O(1)
$$

The CKM matrix is in fact described by 4 real parameters, one of which is this phase (see e.g. the conventional Chau-Kieung parameterisation [30]). Its modulus, $\left|V_{\text {ckm }}\right|$, is therefore described by 3 real parameters which we take to be (in the notation of (7.4)) $V_{u s}, V_{c b}$ and $V_{u b}$. At this point, then, we have 11 algebraic expressions for known quantities:

$$
\left\{\begin{array}{l}
m_{i}(\xi) \quad i=u, d, \epsilon, c, s, \mu, b, \tau \\
V_{u s}(\xi), V_{c b}(\xi), V_{u b}(\xi)
\end{array}\right.
$$

We now respectively denote these by $f_{i}(\xi)$ and the corresponding data pieces by $E_{i}$ $(i=1, \ldots, 11)$. We also have the expression (from (5.1) and (5.2)):

$$
m_{t} \simeq\left|\gamma_{33}^{U}\right|\langle\phi\rangle_{\mathrm{ws}}
$$

so that $\left|\gamma_{33}^{U}\right|$ is a free parameter in our analysis which we can:

(a) use to fix the top mass by hand; or

(b) incorporate in our fitting procedure so that the "most favourable" top mass is predicted for each model.

In any event, we absorb $\left|h_{33}^{L}\right|$ into $\langle\phi\rangle_{w s}$ so that all masses are now predicted in units of $m_{t}$.

(iii) We now construct a penalty function, $x^{2}(\xi)$, whose minimum will indicate the best fit possible of a particular ansatz to the data. Firstly, as our procedure is only designed to fit the data up to numerical factors, we demand that a prediction which is inaccurate by a factor $r$ receives the same penalty as one which is inaccurate by a factor of $1 / r$, where $r$ is some real number. In addition, we quantify our oft-repeated statement that 
our predictions are accurate only up to factors of $O(1)$ by assuming that the error on each prediction $f_{i}$ is a factor of e. This is numerically convenient, but it should be borne in mind that it is perhaps too strict since we are only interested in order of magnitude features. Should some predictions creep outside this assumed error, the model in question should not necessarily be dismissed - our models should live or die by their order of magnitude predictions. Note further that this "theoretical" error on the $f_{i}$ completely swamps the "experimental" error on the $E_{i}$. We choose the simplest function which meets our requirements:

$$
\chi^{2}=\sum_{i=1}^{11}\left[\log f_{i}(\xi)-\log E_{i}\right]^{2}
$$

(iv) Finally, we minimise this function for any particular model and ansatz using a routine from the NAG library and run our $M_{P}$ results back down to $1 \mathrm{GeV}$ for presentation here.

\section{A Diagonalisation Example}

In order to elucidate further on our treatment of the mass matrices, in this section we explicitly diagonalise a typical mass matrix. We choose $M_{D}$ in the $H_{1} \times U(1)_{f}$ model and adopt the product ansatz. Using (5.22), (6.26) and including the $\gamma_{i j}$ of (5.2) so that our approach is completely clear, we find:

$$
M_{D}=\left(\begin{array}{ccc}
\gamma_{11} \lambda_{1}^{3} & \gamma_{12} \lambda_{1}^{2} \epsilon_{2} \lambda_{f} & \gamma_{13} \lambda_{1}^{2} \lambda_{f} \\
\gamma_{21} \lambda_{1} \lambda_{f}^{3} & \gamma_{22} \epsilon_{2} \lambda_{f}^{2} & \gamma_{23} \lambda_{f}^{4} \\
\gamma_{31} \lambda_{1} \lambda_{f}^{3} & \gamma_{32} \epsilon_{2} \lambda_{f}^{4} & \gamma_{33} \lambda_{f}^{2}
\end{array}\right)
$$

We should really write $\gamma_{i j}^{D}$ but we suppress the superscript. Then to leading order (with

$$
\left.\gamma_{i j}^{2} \equiv \gamma_{i j}^{*} \gamma_{i j}\right): \text { : } M_{D}^{\dagger} M_{D}=\left(\begin{array}{ccc}
\left(\gamma_{21}^{2}+\gamma_{31}^{2}\right) \lambda_{1}^{2} \lambda_{f}^{6}+\gamma_{11}^{2} \lambda_{1}^{6} & \gamma_{21}^{*} \gamma_{22} \lambda_{1} \epsilon_{2} \lambda_{f}^{5} & \gamma_{31}^{*} \gamma_{33} \lambda_{1} \lambda_{f}^{5} \\
\gamma_{21} \gamma_{22}^{*} \lambda_{1} \epsilon_{2} \lambda_{f}^{5} & \gamma_{22}^{2} \epsilon_{2}^{2} \lambda_{f}^{4} & \left(\gamma_{22}^{*} \gamma_{23}+\gamma_{32}^{*} \gamma_{33}\right) \epsilon_{2} \lambda_{f}^{6} \\
\gamma_{31} \gamma_{33}^{*} \lambda_{1} \lambda_{f}^{5} & \left(\gamma_{22} \gamma_{23}^{*}+\gamma_{32} \gamma_{33}^{*}\right) \epsilon_{2} \lambda_{f}^{6} & \gamma_{33}^{2} \lambda_{f}^{4}
\end{array}\right)
$$


The $\gamma_{11}^{2}$ term in the (1,1) entry has been retained in order to ensure that the leading order term in $\operatorname{det}\left(M_{D}^{\dagger} M_{D}\right)$ is correctly seen to be $\gamma_{11}^{2} \hat{\gamma}_{22}^{2} \gamma_{33}^{2} \lambda_{1}^{6} \epsilon_{2}^{2} \lambda_{f}^{8}$.

Since we "know" a priori that the eigenvalues of this matrix must satisfy:

$$
m_{d}^{2} \ll m_{s}^{2} \ll m_{b}^{2}
$$

we can make the simplifying approximations:

$$
\begin{aligned}
m_{b}^{2} & \simeq \text { leading term in } M I_{D}^{\dagger} M_{D} \\
m_{s}^{2} m_{b}^{2} & \simeq \text { leading term in minors of } M_{D}^{\dagger} M_{D} \\
m_{d}^{2} m_{s}^{2} m_{b}^{2} & \simeq \text { leading term in } \operatorname{det}\left(M_{D}^{\dagger} M_{D}\right)
\end{aligned}
$$

which are entirely consistent with our aim of pinning down the masses order of magnitude wise. Analagous approximations are made for all mass matrices throughout this paper. These immediately yield:

$$
\begin{aligned}
& m_{b}^{2} \simeq \gamma_{33}^{2} \lambda_{f}^{4} \\
& m_{s}^{2} \simeq \gamma_{22}^{2} \epsilon_{2}^{2} \lambda_{f}^{4} \\
& m_{d}^{2} \simeq \gamma_{11}^{2} \lambda_{1}^{6}
\end{aligned}
$$

providing $M_{D}(2,1)<M_{D}(2,2)$ i.e. providing $\lambda_{1} \lambda_{f}<\epsilon_{2}$. (Different expressions for $m_{s}$ and $m_{d}$ are easily calculated in the region of parameter space where $\lambda_{1} \lambda_{f}>\epsilon_{2}$ ). Calculating the eigenvectors corresponding to these eigenvalues gives the unitary matrix $R_{D}$ of (7.6) to be:

$$
R_{D} \simeq\left(\begin{array}{ccc}
1 & \frac{\gamma_{21}^{*}}{\gamma_{22}^{*}} \frac{\lambda_{1} \lambda_{f}}{\epsilon_{2}} & \frac{\gamma_{31}^{*}}{\gamma_{33}^{*}} \lambda_{1} \lambda_{f} \\
-R_{D}(1,2) & 1 & \frac{\gamma_{22}^{*} \gamma_{23}+\gamma_{32}^{*} \gamma_{33}}{\gamma_{33}^{2}} \epsilon_{2} \lambda_{f}^{2} \\
-R_{D}(1,3) & -R_{D}(2,3)-R_{D}(1,2) R_{D}(1,3) & 1
\end{array}\right)
$$

Again, we have kept only the leading order terms (it is not algebraically clear which of the two terms in $R_{D}(3,2)$ is dominant). Note that $R_{D}$ will be different in the region of parameter space where $\lambda_{1} \lambda_{f}>\epsilon_{2}$.

The contribution of the mass predictions of $(8.5)$ to the $\chi^{2}$ function is now very easy to construct. We have, in the notation of (7.11) with the $O(1) \gamma_{i j}$ now dropped: 


$$
\begin{aligned}
& f_{2}(\xi) \equiv m_{d}=\lambda_{1}^{3} \\
& f_{5}(\xi) \equiv m_{s}=\epsilon_{2} \lambda_{f}^{2} \\
& f_{7}(\xi) \equiv m_{b}=\lambda_{f}^{2}
\end{aligned}
$$

The reader is reminded that all masses are understood to be in units of $m_{t}$. The mixing angle contributions are more problematic because we have to consider $R_{U}^{\dagger} R_{D}$ and not just $R_{D}$. But it is a simple matter to show that $R_{U}=R_{D}$ after the transformation $\gamma_{i j}^{D} \rightarrow \gamma_{i j}^{U}$ for all $i$ and $j$ i.e. the matrices are identical order of magnitude wise. It then follows that:

$$
\begin{aligned}
f_{9}(\xi) & \equiv V_{u s}=\lambda_{1} \lambda_{f} / \epsilon_{2} \\
f_{10}(\xi) & \equiv V_{c b}=\max \left(\epsilon_{2} \lambda_{f}^{2}, \lambda_{1}^{2} \lambda_{f}^{2} / \epsilon_{2}\right) \\
f_{11}(\xi) & \equiv V_{u b}=\lambda_{1} \lambda_{f}
\end{aligned}
$$

The eigenvalues of $M_{L^{i}}^{\dagger} M_{L^{i}}$ and $M_{l}^{\dagger} M_{l}$ give the remaining $f_{i}(\xi)$ in an obvious manner, so that the complete $\chi^{2}$ function is determined for the $H_{1} \times U(1)_{f}$ model with the product ansatz.

\section{Results}

Note that, for all models considered here. the fit for the product ansatz uses 3 parameters, that for the mixed ansatz uses 4 while the fit for the metric ansatz uses 6 .

9.1. $H_{1} \times U(1)_{f}$

In this model the PCCSs are $U_{1}(1) \times S U_{2}(2) \times U(1)_{f}$ and the $U(1)_{f}$ charges of $(6.26)$ constitute an anomaly-free set when the full gauge group is $S U(3) \times S U_{2}(2) \times S U_{13}(2) \times$ $U_{1}(1) \times U_{23}(1) \times U(1)_{f}$. In fact, the full gauge group can be as large as $S M G_{1} \times S U_{23}(3) \times$ $S U_{2}(2) \times S U_{3}(2) \times U_{23}(1) \times U(1)_{f}$ and still remain free of anomalies, courtesy of the fact 
that the 1 st generation states have zero $U(1)_{f}$ charge. We now examine this model using all 3 ansätze of Section 5 .

\subsubsection{Product Ansatz}

Using equations (5.22) and (6.26), the mass matrices are easily seen to be:

$$
\begin{gathered}
M_{U} \simeq\left(\begin{array}{ccc}
\lambda_{1}^{3} & \lambda_{1}^{4} \epsilon_{2} \lambda_{f} & \lambda_{1}^{4} \lambda_{f} \\
\lambda_{1} \lambda_{f} & \epsilon_{2} & \lambda_{f}^{2} \\
\lambda_{1} \lambda_{f} & \epsilon_{2} \lambda_{f}^{2} & 1
\end{array}\right) \\
M_{D} \simeq\left(\begin{array}{ccc}
\lambda_{1}^{3} & \lambda_{1}^{2} \epsilon_{2} \lambda_{f} & \lambda_{1}^{2} \lambda_{f} \\
\lambda_{1} \lambda_{f}^{3} & \epsilon_{2} \lambda_{f}^{2} & \lambda_{f}^{4} \\
\lambda_{1} \lambda_{f}^{3} & \epsilon_{2} \lambda_{f}^{4} & \lambda_{f}^{2}
\end{array}\right) \\
M_{l} \simeq\left(\begin{array}{ccc}
\lambda_{1}^{3} & \lambda_{1}^{6} \epsilon_{2} \lambda_{f}^{3} & \lambda_{1}^{6} \lambda_{f}^{3} \\
\lambda_{1}^{3} \lambda_{f} & \epsilon_{2} \lambda_{f}^{2} & \lambda_{f}^{4} \\
\lambda_{1}^{3} \lambda_{f} & \epsilon_{2} \lambda_{f}^{4} & \lambda_{f}^{2}
\end{array}\right)
\end{gathered}
$$

Our algebraic $M_{P}$-scale predictions are then (in a very wide region of parameter space):

$$
\begin{array}{ll}
m_{\tau} \simeq M_{l}(3,3) \simeq \lambda_{f}^{2}, & m_{\mu} \simeq M_{l}(2,2) \simeq \epsilon_{2} \lambda_{f}^{2}, \quad m_{e} \simeq M_{l}(1,1) \simeq \lambda_{1}^{3} \\
m_{b} \simeq M_{D}(3,3) \simeq \lambda_{f}^{2}, & m_{s} \simeq M_{D}(2,1) \simeq \lambda_{1} \lambda_{f}^{3}, \quad m_{d} \simeq \frac{M_{D}(1,1) M_{D}(2,2)}{M_{D}(2,1)} \simeq \frac{\lambda_{1}^{2} \epsilon_{2}}{\lambda_{f}} \\
m_{t} \simeq 1 & m_{c} \simeq M_{U^{\prime}}(2,1) \simeq \lambda_{1} \lambda_{f}, \quad m_{u} \simeq \frac{M_{U^{\prime}}(1,1) M_{U^{\prime}}(2,2)}{M_{U}(2,1)} \simeq \frac{\lambda_{1}^{2} \epsilon_{2}}{\lambda_{f}}
\end{array}
$$

and:

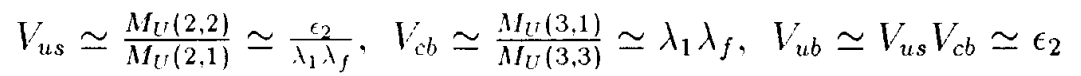

We construct the $\lambda^{2}$ function as shown previously, and find the values of $\left(\lambda_{1}, \epsilon_{2}, \lambda_{f}\right)$ which minimise it. Results are shown in Table 2 for $m_{t}=100$ and $200 \mathrm{GeV}$ respectively (given a free choice. $m_{t}$ will lie at $100 \mathrm{GeV}$, the lower end of its allowed range). The first point to note is that, order of magnitude wise, these results are good for the low top mass but are less impressive for the high top mass. Nevertheless, algebraic order of magnitude predictions such as $V_{u b} \simeq V_{u s} V_{c b}$ are excellent. We draw attention to some minor problems: 


\section{Table 2}

\begin{tabular}{|c|c|c|}
\hline \multirow{2}{*}{$\begin{array}{c}\text { Fit } \\
\text { Results }\end{array}$} & \multicolumn{2}{|c|}{$H_{1} \times U(1)_{f}:$ Product Ansatz } \\
\hline & $m_{t}^{\text {phys }}=100 \mathrm{G} \in \mathrm{V}$ & $m_{t}^{\text {phys }}=200 \mathrm{G \epsilon V}$ \\
\hline$x^{2}$ & 8.9 & 14 \\
\hline$\lambda_{1}$ & 0.036 & 0.023 \\
\hline$\epsilon_{2}$ & 0.0076 & 0.0041 \\
\hline$\lambda_{f}$ & 0.34 & 0.22 \\
\hline$m_{\epsilon}(\mathrm{MeV})$ & 1.6 & 1.5 \\
\hline$m_{\mu}(\mathrm{MeV})$ & 28 & 26 \\
\hline$m_{\tau}(\mathrm{GeV})$ & 3.7 & 6.3 \\
\hline$m_{d}(\mathrm{MeV})$ & 4.7 & 5.9 \\
\hline$m_{s}(\mathrm{MeV})$ & 220 & 160 \\
\hline$m_{b}^{p h y s}(\mathrm{GeV})$ & 12 & 20 \\
\hline$m_{u}(\mathrm{MeV})$ & 4.7 & 5.9 \\
\hline$m_{c}^{\text {phys }}(\mathrm{GeV})$ & 1.9 & 2.8 \\
\hline$V_{u s}$ & 0.53 & 0.62 \\
\hline$V_{c b}$ & 0.012 & 0.0052 \\
\hline$V_{u b}$ & 0.0076 & 0.0041 \\
\hline
\end{tabular}

Results of a $x^{2}$-fit to fermion masses and mixing angles for $H_{1} \times U(1)_{f}$ with the product ansatz. All masses are running masses evaluated at $1 \mathrm{GeV}$ unless otherwise stated.

(i) the large $m_{b}$. This is a result of the $M_{P}$-scale prediction:

$$
m_{b} / m_{t} \simeq m_{s} / m_{c},
$$

a relation which gets less realistic as $m_{t}$ rises;

(ii) the large $m_{\tau}$. This is a result of the $M_{P}$-scale prediction:

$$
m_{b} \simeq m_{\tau}
$$

and the large $m_{b}$, and is again more pronounced at high $m_{t}$; 
(iii) the small $m_{\mu}$ and high $V_{u s}^{r}$. These are results of the $M_{P^{-s c a l e}}$ prediction:

$$
V_{u s} \simeq m_{\mu} / m_{s}
$$

and the contrasting running of $V_{u s} . m_{\mu}$ and $m_{s}$ between $M_{P}$ and $1 \mathrm{GeV}\left(V_{u s}\right.$ is practically constant, $m_{\mu}$ runs by about $10 \%$ and $m_{s}$ by a factor of $5-6$ [27]). Evolving the variables in (9.6) down to $1 \mathrm{GeV}$ and rearranging gives (approximately):

$$
m_{s} \simeq \frac{5 m_{\mu}}{V_{u s}}
$$

which should be compared with the required relation:

$$
m_{s} \simeq 2 m_{\mu}
$$

The model is thus bound to predict a high $V_{u s}$ and low $m_{\mu}$ ( $m_{s}$ cannot stray too high due to $(9.4))$.

(iv) the low $V_{c b}$ at $m_{t}=200 \mathrm{GeV}$. This is the only prediction which is clearly unacceptable, even order of magnitude wise. It is a result of the $M_{P}$-scale relation:

$$
V_{c b} \simeq m_{c} / m_{t}
$$

which is obviously bad for high $m_{t}$.

\subsubsection{Mixed Ansatz}

Using equations (5.2:3) and (6.26), the mass matrices are given by:

$$
\begin{aligned}
& M_{U} \simeq\left(\begin{array}{ccc}
\mathrm{e}^{-\sqrt{g_{11}}} & \epsilon_{2} \mathrm{e}^{-\sqrt{16 g_{11}+g_{f f}+8 g_{1 f}}} & \mathrm{e}^{-\sqrt{16 g_{11}+g_{f f}-8 g_{1 f}}} \\
\mathrm{e}^{-\sqrt{g_{11}+g_{f f}+2 g_{1 f}}} & \epsilon_{2} & \mathrm{e}^{-\sqrt{4 g_{f f}}} \\
\mathrm{e}^{-\sqrt{g_{11}+g_{f f}-2 g_{1 f}}} & \epsilon_{2} \mathrm{e}^{-\sqrt{4 g_{f f}}} & 1
\end{array}\right) \\
& M_{D} \simeq\left(\begin{array}{ccc}
\mathrm{e}^{-\sqrt{9 g_{11}}} & \epsilon_{2} \mathrm{e}^{-\sqrt{4 g_{11}+g_{f f}-4 g_{1 f}}} & \mathrm{e}^{-\sqrt{4 g_{11}+g_{f f}+4 g_{1 f}}} \\
\mathrm{e}^{-\sqrt{g_{11}+g_{f f}+6 g_{1 f}}} & \epsilon_{2} \mathrm{e}^{-\sqrt{4 g_{f f}}} & \mathrm{e}^{-\sqrt{16 g_{f f}}} \\
\mathrm{e}^{-\sqrt{g_{11}+g_{f f}-6 g_{1 f}}} & \epsilon_{2} \mathrm{e}^{-\sqrt{16 g_{f f}}} & \mathrm{e}^{-\sqrt{4 g_{f f}}}
\end{array}\right) \\
& M_{l} \simeq\left(\begin{array}{ccc}
\mathrm{e}^{-\sqrt{9_{g_{11}}}} & \epsilon_{2} \mathrm{e}^{-\sqrt{36 g_{11}+9 g_{f f}+36 g_{1 f}}} & \mathrm{e}^{-\sqrt{36 g_{11}+9 g_{f f}-36 g_{1 f}}} \\
\mathrm{e}^{-\sqrt{9 g_{11}+g_{f f}+6 g_{1 f}}} & \epsilon_{2} \mathrm{e}^{-\sqrt{4 g_{f f}}} & \mathrm{e}^{-\sqrt{16 g_{f f}}} \\
\mathrm{e}^{-\sqrt{9 g_{11}+g_{f f}-6 g_{1 f}}} & \epsilon_{2} \mathrm{e}^{-\sqrt{16 g_{f f}}} & \mathrm{e}^{-\sqrt{4 g_{f f}}}
\end{array}\right)
\end{aligned}
$$


Then our algebraic $M_{P}$-scale predictions are (in a wide region of parameter space):

$$
\begin{array}{ll}
m_{\tau} \simeq M_{l}(3,3), \quad m_{u} \simeq M_{l}(2,2), \quad m_{e} \simeq M_{l}(1,1) \\
m_{b} \simeq M_{D}(3,3), & m_{s} \simeq M_{D}(2,1), \quad m_{d} \simeq M_{D}(1,2) \\
m_{t} \simeq 1 & m_{c} \simeq M_{U^{\prime}}(2,1), \quad m_{u} \simeq \frac{M_{L^{\prime}}(1,1) M_{U^{\prime}}(2,2)}{M_{U^{\prime}}(2,1)}
\end{array}
$$

and:

$$
V_{u s} \simeq \frac{M_{U^{*}}(2,2)}{M_{U}(2,1)}, \quad V_{c b} \simeq \frac{M_{U^{\prime}}(3.1)}{M_{U}(3.3)}, \quad V_{u b} \simeq V_{u s} V_{c b}
$$

Note that several matrix elements are actually algebraically equivalent to the corresponding elements in (9.1) with the natural identification of (5.25). Those which contribute to $\chi^{2}$ and are inequivalent are $M_{L, D}(2,1), M_{I, D}(3,1)$ and $M_{D}(1,2)$.

Consider the down quark matrix. As before we have the algebraic predictions:

$$
\begin{aligned}
m_{s} & \simeq M_{D}(2,1) \\
V_{u b} & \simeq\left[\frac{V_{u s}}{M_{D}(3,3)}\right] M_{D}(3,1)
\end{aligned}
$$

In the product ansatz results $m_{s}$ and $V_{u b}$ were both good, although $V_{u b}$ was a little high. But now the contrasting contributions of $g_{1 f}$ to $M_{D}(2,1)$ and $M_{D}(3,1)$ cause problems. For example, in order to algebraically hold $M_{D}(2,1)$ as low as it was previously we would have to choose $g_{1 f}$ to lie on its upper limit:

$$
g_{1 f}=\sqrt{g_{11} g_{f f}}
$$

(constraints on the metric parameters are easily derived from the fact that $\mathcal{G}$ must be positive semi-definite). But then $M_{D}(3,1)$ would be much larger than it was previously, causing $V_{u b}$ to be too large. Conversely, algebraically holding $M_{D}(3,1)$ as low as its previous value requires that $g_{1 f}$ lie on its lower limit:

$$
g_{1 f}=-\sqrt{g_{11} g_{f f}}
$$

But then $M_{D}(2,1)$ (and hence $m_{s}$ ) becomes very large. The $\chi^{2}$ minima shown in Table 3 represent a balance in this conflict, with $m_{s}$ and $V_{u b}$ both fairly high. A similar problem exists in $M_{U}$ with the charm mass.

So the mixed ansatz results are broadly similar to those of the product ansatz, with some of the same minor problems (high $V_{u s}$, low $m_{\mu}$ ), except for a more serious problem 


\begin{tabular}{||c||c|c||}
\hline \multirow{2}{*}{$\begin{array}{c}\text { Fit } \\
\text { Results }\end{array}$} & \multicolumn{2}{|c||}{$H_{1} \times U(1)_{f}:$ Mixed Ansatz } \\
\cline { 2 - 3 }$m_{t}^{\text {phys }}=100 \mathrm{GeV}$ & $m_{t}^{\text {phys }}=200 \mathrm{GeV}$ \\
\hline \hline$\lambda^{2}$ & 15 & 17 \\
\hline \hline$\epsilon_{2}$ & 0.022 & 0.012 \\
\hline$g_{11}$ & 11.3 & 14.6 \\
\hline$g_{f f}$ & 2.70 & 4.57 \\
\hline$g_{1 f}$ & 1.45 & 1.21 \\
\hline$m_{\epsilon}(\mathrm{MeV})$ & 1.3 & 1.3 \\
\hline$m_{\mu}(\mathrm{MeV})$ & 26 & 22 \\
\hline$m_{\tau}(\mathrm{GeV})$ & 1.2 & 1.7 \\
\hline \hline$m_{d}(\mathrm{MeV})$ & 2.2 & 2.0 \\
\hline$m_{s}(\mathrm{MeV})$ & 880 & 570 \\
\hline$m_{b}^{\text {phys }}(\mathrm{GeV})$ & 4.7 & 6.4 \\
\hline \hline$m_{u}(\mathrm{MeV})$ & 4.0 & 4.7 \\
\hline$m_{c}^{\text {phys }}(\mathrm{GeV})$ & 4.5 & 7.4 \\
\hline \hline$V_{u s}$ & 0.52 & 0.60 \\
\hline$V_{c b}$ & 0.034 & 0.026 \\
\hline$V_{u b}$ & 0.021 & 0.019 \\
\hline
\end{tabular}

Table 3

Results of a $\chi^{2}$-fit to fermion masses and mixing angles for $H_{1} \times U(1)_{f}$ with the mixed ansatz. All masses are running masses evaluated at $1 \mathrm{GeV}$ unless otherwise stated.

in the $m_{c}-m_{s}-V_{u b}$ sector. This is the main reason for the deterioration in $\chi^{2}$ in going from the product to the mixed ansatz. Given a free choice, the top mass will lie at 100 $\mathrm{GeV}$. 


\subsection{Metric Ansat:}

To facilitate presentation of the mass matrices here, we introduce 3 matrices $N_{U}, N_{D}$ and $N_{l}$ whose elements are the squares of the exponents of the matrix elements defined in (5.24). That is $N_{U}(i, j)=\left(\log \left[M_{U^{\prime}}(i, j)\right]\right)^{2}$ etc and so, using equations (5.24) and (6.26), we have:

$$
\begin{aligned}
& N_{U} \simeq\left(\begin{array}{ccc}
9 g_{11} & 16 g_{11}+g_{22}+g_{f f} & 16 g_{11}+g_{f f}-8 g_{1 f} \\
& -8 g_{12}+8 g_{1 f}-2 g_{2 f} & \\
g_{11}+g_{f f}+2 g_{1 f} & g_{22} & 4 g_{f f} \\
& & 0
\end{array}\right) \\
& N_{D} \simeq\left(\begin{array}{ccc}
9 g_{11} & 4 g_{11}+g_{22}+g_{f f} & 4 g_{11}+g_{f f}+4 g_{1 f} \\
& -4 g_{12}-4 g_{1 f}+2 g_{2 f} \\
& \\
g_{11}+9 g_{f f}+6 g_{1 f} & g_{22}+4 g_{f f}-4 g_{2 f} & 16 g_{f f} \\
& \\
g_{11}+9 g_{f f}-6 g_{1 f} & g_{22}+16 g_{f f}+8 g_{2 f} & 4 g_{f f}
\end{array}\right) \\
& N_{l} \simeq\left(\begin{array}{ccc}
9 g_{11} & 36 g_{11}+g_{22}+9 g_{f f} & 36 g_{11}+9 g_{f f}-36 g_{1 f} \\
& -12 g_{12}+36 g_{1 f}-6 g_{2 f} & \\
& \\
9 g_{11}+g_{f f}+6 g_{1 f} & g_{22}+4 g_{f f}-4 g_{2 f} & 16 g_{f f} \\
& & \\
9 g_{11}+g_{f f}-6 g_{1 f} & g_{22}+16 g_{f f}-8 g_{2 f} & 4 g_{f f}
\end{array}\right)
\end{aligned}
$$


With this ansatz, our algebraic predictions are (in a wide region of parameter space):

$$
\begin{array}{ll}
m_{\tau} \simeq M_{l}(3,3), \quad m_{\mu} \simeq M_{l}(2,2), \quad m_{e} \simeq M_{l}(1,1) \\
m_{b} \simeq M_{D}(3,3), & m_{s} \simeq M_{D}(2,1), \quad m_{d} \simeq M_{D}(1,2) \\
m_{t} \simeq 1 & m_{c} \simeq M_{U^{\prime}}(2,1), \quad m_{u} \simeq \frac{M_{U^{*}}(1.1) M_{U^{\prime}}(2.2)}{M_{U^{\prime}}(2,1)}
\end{array}
$$

and:

$$
V_{u s} \simeq \frac{M_{D}(2,2)}{M_{D}(2,1)}, \quad V_{c b} \simeq \frac{M_{D}(3,1)}{M_{D}(3,3)}, \quad V_{u b} \simeq \frac{M_{D}(3,2)}{M_{D}(3,3)}
$$

Results of the 6 parameter $\chi^{2}$ fit are shown in Table 4 for $m_{t}=100$ and $200 \mathrm{GeV}$ respectively. Given a free choice, $m_{t}$ will lie at $104 \mathrm{GeV}$ and the results are almost identical to the first column of Table 4. That the top mass naturally lies in the region $100-200 \mathrm{GeV}$ is an attractive feature here. Far from continuing the deterioration initiated by the mixed ansatz, these fits have the lowest $\lambda^{2}$ shown so far. With the mixed ansatz, the easing of suppression on $M_{U, D}(2,1)$ and $M_{U, D}(3,1)$ without affecting $M_{U, D}(2,2)$ caused problems; now, $M_{U, D}(2,2)$ and $M_{U, D}(3,2)$ are also inequivalent to the corresponding elements in (9.1) and this leads to the improvement. The conflict between $M_{D}(2,1)$ and $M_{D}(3,1)$ still exists, but we are no longer burdened by the prediction:

$$
V_{u b} \simeq\left[\frac{V_{u s}}{M_{D}(3,3)}\right] M_{D}(3,1)
$$

instead finding that:

$$
V_{u b} \simeq \frac{M_{D}(3,2)}{M_{D}(3,3)}
$$

due to the easing of suppression on $M_{D}(3,2)$. We can hence again obtain good values for $m_{s}$ and $V_{u b}$.

The improvement in $x^{2}$ from the product ansatz is largely because of:

(i) the fact that det $M_{D}\left(=m_{d} m_{s} m_{b}\right)$ is now bigger than its previous value of $M_{D}(1,1) M_{D}(2,2) M_{D}(3,3)$, allowing $m_{d}$ to assume a larger, more realistic value;

(ii) and the rise in $V_{c b}$. A prediction for all ansätze has been:

$$
V_{c b} \simeq \frac{M_{D}(3,1)}{M_{D}(3.3)}
$$

and the metric ansatz caters for a larger $M_{D}(3,1)$ than the product ansatz. 


\begin{tabular}{|c|c|c|}
\hline \multirow{2}{*}{$\begin{array}{c}\text { Fit } \\
\text { Results }\end{array}$} & \multicolumn{2}{|c|}{$H_{1} \times U(1)_{f}:$ Metric Ansatz } \\
\hline & $m_{t}^{p h y s}=100 \mathrm{GeV}$ & $m_{t}^{p h y s}=200 \mathrm{GeV}$ \\
\hline$\lambda^{2}$ & 8.0 & 8.8 \\
\hline$g_{11}$ & 11.8 & 15.4 \\
\hline$y_{22}$ & 24.8 & 30.4 \\
\hline$g_{f f}$ & 3.05 & 5.05 \\
\hline$g_{12}$ & -4.34 & -7.40 \\
\hline$y_{1 f}$ & 1.06 & 0.763 \\
\hline$g_{2 f}$ & 0.872 & 3.19 \\
\hline$m_{\epsilon}(\mathrm{MeV})$ & 1.1 & 0.97 \\
\hline$m_{\mu}(\mathrm{MeV})$ & 56 & 44 \\
\hline$m_{\tau}(\mathrm{GeV})$ & 0.98 & 1.4 \\
\hline$m_{d}(\mathrm{MeV})$ & 9.2 & 9.2 \\
\hline$m_{s}(\mathrm{MeV})$ & 510 & 340 \\
\hline$m_{b}^{\text {phys }}(\mathrm{GeV})$ & 4.0 & 5.3 \\
\hline$m_{u}(\mathrm{MeV})$ & 1.3 & 1.5 \\
\hline$m_{c}^{\text {phys }}(\mathrm{GeV})$ & 3.7 & 6.0 \\
\hline$V_{u s}$ & 0.47 & 0.53 \\
\hline$V_{c b}$ & 0.038 & 0.028 \\
\hline$V_{u b}$ & 0.0094 & 0.0086 \\
\hline
\end{tabular}

Table 4

Results of a $\chi^{2}$-fit to fermion masses and mixing angles for $H_{1} \times U(1)_{f}$ with the metric ansatz. All masses are running masses evaluated at $1 \mathrm{GeV}$ unless otherwise stated.

\subsubsection{Comments}

Of course, the different ansätze reflect our ignorance of the symmetry breaking process which yields the $S M G$. and so the spread of results across the ansätze can be thought of as some indication of the error in the model results. Overall, then, the PCCSs $U_{1}(1) \times$ 
$S U_{2}(2) \times U(1)_{f}$ are seen to be fairly successful. achieving our aim of accounting for the prominent order of magnitude features of the fermion mass and mixing hierarchies.

\section{3. $H_{2} \times U(1)_{f}$}

Here, the PCCSs are $U_{1}(1) \times U_{2}(1) \times U(1)_{f}$ and the $U(1)_{f}$ charges of $(6.36)$ and $(6.37)$ constitute anomaly-free charge sets when the full gauge group is $S U(3) \times S U(2) \times U_{1}(1) \times$ $U_{2}(1) \times U_{3}(1) \times U(1)_{f}$. Again, as the 1st generation states have zero charge, the full gauge group can be as large as $S M G_{1} \times S U_{23}(3) \times S U_{23}(2) \times U_{2}(1) \times U_{3}(1) \times U(1)_{f}$ and still remain anomaly-free. We now examine both charge sets using the product and mixed ansätze (the metric ansatz is inapplicable as all PCCSs in this model are abelian).

\subsubsection{1st Charge Set: Product Ansatz}

We will in fact show that the charge set of $(6.36)$ is not useful as far as far as generating the mass hierarchy is concerned. With the product ansatz, the quark mass matrices are given by (from equation (5.22)):

$$
\begin{aligned}
& M_{U^{I}} \simeq\left(\begin{array}{ccc}
\lambda_{1}^{3} & \lambda_{1}^{4} \lambda_{2} \lambda_{f}^{5} & \lambda_{1}^{4} \lambda_{f}^{5} \\
\lambda_{1} \lambda_{2}^{4} \lambda_{f}^{5} & \lambda_{2}^{3} & \lambda_{2}^{4} \lambda_{f}^{10} \\
\lambda_{1} \lambda_{f}^{5} & \lambda_{2} \lambda_{f}^{10} & 1
\end{array}\right) \\
& M_{D} \simeq\left(\begin{array}{ccc}
\lambda_{1}^{3} & \lambda_{1}^{2} \lambda_{2} \lambda_{f}^{5} & \lambda_{1}^{2} \lambda_{f}^{5} \\
\lambda_{1} \lambda_{2}^{2} \lambda_{f} & \lambda_{2}^{3} \lambda_{f}^{6} & \lambda_{2}^{2} \lambda_{f}^{4} \\
\lambda_{1} \lambda_{f} & \lambda_{2} \lambda_{f}^{4} & \lambda_{f}^{6}
\end{array}\right)
\end{aligned}
$$

The problem is that we would like to get:

$$
m_{s} \simeq M_{D}(2,2)
$$

but in fact we find that:

$$
m_{s} \simeq \frac{M_{D}(2,3) M_{D}(3,2)}{M_{D}(3,3)}
$$


We then have the unfortunate prediction:

$$
\frac{m_{b}}{m_{t}} \simeq \lambda_{f}^{6} \simeq\left(\frac{m_{s}}{m_{c}}\right)^{3}
$$

which is very bad at both $1 \mathrm{GeV}$ and $M_{P}$.

\subsubsection{1st Charge Set: Metric Ansatz}

We do not give the mass matrices here: the above problem is simply compounded by this less suppressive ansatz. We still predict $(9.24)$ and in fact cannot avoid getting $m_{s}>m_{c}$. We therefore dismiss this charge set as being incompatible with the observed fermion mass hierarchy.

\subsubsection{2nd Charge Set: Product Ansatz}

Using (5.22) and the charge set of (6.37), we find the mass matrices:

$$
\begin{aligned}
& M_{U} \simeq\left(\begin{array}{ccc}
\lambda_{1}^{3} & \lambda_{1}^{4} \lambda_{2} \lambda_{f} & \lambda_{1}^{4} \lambda_{f} \\
\lambda_{1} \lambda_{2}^{4} \lambda_{f} & \lambda_{2}^{3} & \lambda_{2}^{4} \lambda_{f}^{2} \\
\lambda_{1} \lambda_{f} & \lambda_{2} \lambda_{f}^{2} & 1
\end{array}\right) \\
& M_{D} \simeq\left(\begin{array}{ccc}
\lambda_{1}^{3} & \lambda_{1}^{2} \lambda_{2} \lambda_{f} & \lambda_{1}^{2} \lambda_{f} \\
\lambda_{1} \lambda_{2}^{2} \lambda_{f}^{13} & \lambda_{2}^{3} \lambda_{f}^{12} & \lambda_{2}^{2} \lambda_{f}^{14} \\
\lambda_{1} \lambda_{f}^{13} & \lambda_{2} \lambda_{f}^{14} & \lambda_{f}^{12}
\end{array}\right) \\
& M_{l} \simeq\left(\begin{array}{ccc}
\lambda_{1}^{3} & \lambda_{1}^{6} \lambda_{2}^{3} \lambda_{f}^{13} & \lambda_{1}^{6} \lambda_{f}^{13} \\
\lambda_{1}^{3} \lambda_{2}^{6} \lambda_{f} & \lambda_{2}^{3} \lambda_{f}^{12} & \lambda_{2}^{6} \lambda_{f}^{14} \\
\lambda_{1}^{3} \lambda_{f} & \lambda_{2}^{3} \lambda_{f}^{14} & \lambda_{f}^{12}
\end{array}\right)
\end{aligned}
$$

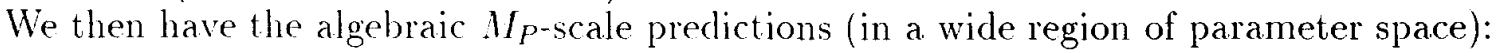

$$
\begin{array}{ll}
m_{\tau} \simeq M_{l}(3,3) \simeq \lambda_{f}^{12}, \quad m_{\mu} \simeq M_{l}(2,2) \simeq \lambda_{2}^{3} \lambda_{f}^{12}, \quad m_{e} \simeq M_{l}(1,1) \simeq \lambda_{1}^{3} \\
m_{b} \simeq M_{D}(3,3) \simeq \lambda_{f}^{12}, & m_{s} \simeq M_{D}(2,2) \simeq \lambda_{2}^{3} \lambda_{f}^{12}, \\
m_{t} \simeq 1 & m_{d} \simeq M_{D}(1,1) \simeq \lambda_{1}^{3} \\
m_{c} \simeq M_{U^{\prime}}(2,2) \simeq \lambda_{2}^{3}, & m_{u} \simeq M_{U}(1,1) \simeq \lambda_{1}^{3}
\end{array}
$$

and:

$$
V_{u s} \simeq \frac{M_{D}(2.1)}{M_{D}(2,2)} \simeq \frac{\lambda_{1} \lambda_{f}}{\lambda_{2}}, \quad V_{c b} \simeq \frac{M I_{D}(3,2)}{M_{D}(3,3)} \simeq \lambda_{2} \lambda_{f}^{2}, \quad V_{u b} \simeq \frac{M_{D}(3,1)}{M_{D}(3,3)} \simeq \lambda_{1} \lambda_{f}
$$


Table 5

\begin{tabular}{||c||c|c||}
\hline \multirow{2}{*}{$\begin{array}{c}\text { Fit } \\
\text { Results }\end{array}$} & \multicolumn{2}{|c||}{$H_{2} \times U(1)_{f}:$ Product Ansatz } \\
\cline { 2 - 3 }${ }^{p h y s}=100 \mathrm{GeV}$ & $m_{t}^{p h y s}=200 \mathrm{GeV}$ \\
\hline \hline$\lambda_{1}$ & 10 & 11 \\
\hline$\lambda_{2}$ & 0.030 & 0.020 \\
\hline$\lambda_{f}$ & 0.25 & 0.20 \\
\hline \hline$m_{\epsilon}(\mathrm{MeV})$ & 0.80 & 0.74 \\
\hline$m_{\mu}(\mathrm{MeV})$ & 0.86 & 0.94 \\
\hline$m_{\tau}(\mathrm{GeV})$ & 35 & 26 \\
\hline$m_{d}(\mathrm{MeV})$ & 2.4 & 3.5 \\
\hline$m_{s}(\mathrm{MeV})$ & 4.2 & 4.6 \\
\hline$m_{b}^{p h y s}(\mathrm{GeV})$ & 170 & 130 \\
\hline \hline$m_{u}(\mathrm{MeV})$ & 8.4 & 12 \\
\hline$m_{c}^{p h_{u s}(\mathrm{GeV})}$ & 4.2 & 0.11 \\
\hline \hline$V_{u s}$ & 2.2 & 3.8 \\
\hline$V_{c b}$ & 0.096 & 0.074 \\
\hline$V_{u b}$ & 0.024 & 0.16 \\
\hline
\end{tabular}

Results of a $\chi^{2}$-fit to fermion masses and mixing angles for $H_{2} \times U(1)_{f}$ (2nd charge set) with the product ansatz. All masses are rumning masses evaluated at $1 \mathrm{GeV}$ unless otherwise stated.

Results of the fit to the values of $\left(\lambda_{1}, \lambda_{2}, \lambda_{f}\right)$ are shown in Table 5 for $m_{t}=100$ and 200 $\mathrm{GeV}$ respectively. Given a free fit to the top mass, the $\chi^{2}$ minimum lies at $m_{t}=116 \mathrm{GeV}$ and the results are almost identical to the first column of Table 5; again, that the top mass naturally lies in the region $100-200 \mathrm{GeV}$ is an attractive feature. The predicted mass hierarchy is good, the $M_{P}$-scale prediction $m_{s} \simeq m_{\mu}$ constitut. g only a small niggle. The mixing hierarchy, however, is noticably poorer than the previous model. The results:

$$
V_{u s}(\text { prediction }) \simeq \frac{1}{2} V_{u s}(\text { actual })
$$


are individually acceptable within our order of magnitude framework, but their combined result

$$
V_{u s}(\text { prediction }) \simeq V_{c b}(\text { prediction })
$$

borders on the unacceptable: we fail to naturally explain the observation $V_{u s} \simeq 5 V_{c b}$. Furthermore, $V_{u b}$ is simply predicted to be 3 times its experimental upper limit, an order of magnitude larger than the value to which we are fitting it. That $V_{c b}$ and $V_{u b}$ are predicted to be too large can be traced to the lack of sufficient suppression of the off-diagonal matrix elements compared to the diagonal elements in $M_{U}$ and $M_{D}$ - a problem not present in the $H_{1} \times U(1)_{f}$ model.

\subsubsection{2nd Charge Set: Mired Ansatz}

Using (5.23), the charge set of (6.37) is seen to yield the mass matrices (again using the matrices $N_{U, D, l}$ to facilitate presentation):

$$
N_{l} \simeq\left(\begin{array}{ccc}
9 g_{11} & 36 g_{11}+9 g_{22}+169 g_{f f} & 36 g_{11}+169 g_{f f}+156 g_{1 f} \\
& -36 g_{12}-156 g_{1 f}+78 g_{2 f} & \\
9 g_{11}+36 g_{22}+g_{f f} & 9 g_{22}+144 g_{f f}-72 g_{2 f} & 36 g_{22}+196 g_{f f}+168 g_{2 f} \\
-36 g_{12}-6 g_{1 f}+12 g_{2 f} & \\
9 g_{11}+g_{f f}+6 g_{1 f} & 9 g_{22}+196 g_{f f}+84 g_{2 f} & 144 g_{f f}
\end{array}\right)
$$




$$
N_{U} \simeq\left(\begin{array}{ccc}
9 g_{11} & 16 g_{11}+g_{22}+g_{f f} & 16 g_{11}+g_{f f}-8 g_{1 f} \\
& -8 g_{12}+8 g_{1 f}-2 g_{2 f} & \\
g_{11}+16 g_{22}+g_{f f} & 9 g_{22} & 16 g_{22}+4 g_{f f}-16 g_{2 f} \\
-8 g_{12}+2 g_{1 f}-8 g_{2 f} & & \\
g_{11}+g_{f f}-2 g_{1 f} & g_{22}+4 g_{f f}-4 g_{2 f} & \\
9 g_{11} & 4 g_{11}+g_{22}+g_{f f} & 4 g_{11}+g_{f f}+4 g_{1 f} \\
N_{D} & +4 g_{12}-4 g_{1 f}-2 g_{2 f} & \\
g_{11}+4 g_{22}+169 g_{f f} & 9 g_{22}+144 g_{f f}+72 g_{2 f} & 4 g_{22}+196 g_{f f}+56 g_{2 f} \\
+4 g_{12}+26 g_{1 f}+52 g_{2 f} & & \\
g_{11}+169 g_{f f}-26 g_{1 f} & g_{22}+196 g_{f f}-28 g_{2 f} & 144 g_{f f}
\end{array}\right)
$$

Our algebraic predictions are then (in a wide region of parameter space):

$$
\begin{array}{ll}
m_{\tau} \simeq M_{l}(3,3), \quad m_{\mu} \simeq M_{l}(2,2), \quad m_{\epsilon} \simeq M_{l}(1,1) \\
m_{b} \simeq M_{D}(3,3), & m_{s} \simeq M_{D}(2.2), \quad m_{d} \simeq \frac{M_{D}(1,2) M_{D}(2,1)}{M_{D}(2,2)} \\
& m_{c} \simeq M_{U}(2,2), \quad m_{u} \simeq M_{U}(1,1)
\end{array}
$$

and:

$$
V_{u s} \simeq \frac{M_{D}(2.1)}{M_{D}(2,2)}, \quad V_{c b} \simeq \frac{M_{D}(3.2)}{M_{D}(3.3)}, \quad V_{u b} \simeq \frac{M_{D}(3.1)}{M_{D}(3.3)}
$$

Results are shown in Table 6 for $m_{t}=100$ and $200 \mathrm{GeV}$ respectively. These predictions are exactly as for the product ansatz, but this in fact causes problems:

(i) the product ansatz relation $m_{s} \simeq m_{\mu}$ is ruined because $g_{2 f}$ contributes to $M_{D}(2,2)$ and $M_{l}(2,2)$ with opposite sign and large coefficient. This drives $m_{s}$ and $m_{\mu}$ apart, the results predicting that $m_{s}$ is an order of magnitude bigger than $m_{\mu}$;

(ii) and the mixing angles are now numerically bigger simply because the mixed ansatz is less suppressive i.e. the problem of large mixing angles discussed above is merely exacer- 


\begin{tabular}{||c||c|c||}
\hline \multirow{2}{*}{$\begin{array}{c}\text { Fit } \\
\text { Results }\end{array}$} & \multicolumn{2}{|c||}{$H_{2} \times L(1)_{f}:$ Mixed Ansatz } \\
\cline { 2 - 3 }$m_{t}^{\text {phys }}=100 \mathrm{GeV}$ & $m_{t}^{\text {phys }}=200 \mathrm{GeV}$ \\
\hline \hline$I^{2}$ & 18.4 & 17.8 \\
\hline$g_{11}$ & 14.7 & 18.3 \\
\hline$g_{22}$ & 3.56 & 4.82 \\
\hline$g_{f f}$ & 0.047 & 0.116 \\
\hline$g_{12}$ & 2.25 & 3.55 \\
\hline$g_{1 f}$ & 0.363 & 0.392 \\
\hline$g_{2 f}$ & 0.111 & 0.036 \\
\hline \hline$m_{e}(\mathrm{MeV})$ & 0.33 & 0.33 \\
\hline$m_{\mu}(\mathrm{MeV})$ & 35 & 45 \\
\hline$m_{\tau}(\mathrm{GeV})$ & 2.4 & 2.1 \\
\hline \hline$m_{d}(\mathrm{MeV})$ & 31 & 33 \\
\hline$m_{s}(\mathrm{MeV})$ & 620 & 310 \\
\hline$m_{b}^{\text {phys }}(\mathrm{GeV})$ & 8.4 & 7.5 \\
\hline \hline$m_{u}(\mathrm{MeV})$ & 1.6 & 1.6 \\
\hline$m_{c}^{\text {phys }}(\mathrm{GeV})$ & 0.76 & 1.0 \\
\hline \hline$V_{u s}$ & 0.18 & 0.19 \\
\hline$V_{c b}$ & 0.24 & 0.27 \\
\hline$V_{u b}$ & 0.046 & 0.056 \\
\hline
\end{tabular}

Table 6

Results of a $\chi^{2}$-fit to fermion masses and mixing angles for $H_{2} \times U(1)_{f}$ (2nd charge set) with the mixed ansatz. All masses are running masses evaluated at $1 \mathrm{GeV}$ unless otherwise stated.

bated when we use the mixed ansatz.

A side effect of these large mixing angles is this ansatz' preference for a large top mass (given a free fit, $m_{t}$ lies at $200 \mathrm{(ieV)}$ ). In an effort to hold down the mixing angles, it is necessary to push $g_{f f}$ as high as possible in order to suppress $M_{D}(3,1)$ and $M_{D}(3,2)$. But 
then the ratio $m_{b} / m_{t}$ is pushed down. hence the preference for a large top mass, so that $m_{b}$ can still lie near its accepted value.

\subsubsection{Comments}

The PCCSs $U_{1}(1) \times U_{2}(1) \times U^{\prime}(1)_{f}$ are not as successful as $U_{1}(1) \times S U_{2}(2) \times U(1)_{f}$, even for the 2 nd charge set. They fail to satisfactorily account for the order of magnitude features of the quark mixing hierarchy because, as we have already said, the off-diagonal elements of the quark mass matrices are not sufficiently suppressed compared to the diagonal ones.

9.4. $H_{3} \times U(1)_{f}$

Here, the PCCSs are $S U_{1}(2) \times S U_{2}(2) \times U(1)_{f}$ and the $U(1)_{f}$ charges of $(6.38)$ constitute an anomaly-free charge set when the full gauge group is $S U(3) \times S U_{1}(2) \times S U_{2}(2) \times$ $S U_{3}(2) \times U(1)_{f}$. As before, the full gauge group can be $S M G_{1} \times S U_{23}(3) \times S U_{2}(2) \times$ $S U_{3}(2) \times U_{23}(1) \times U(1)_{f}$ and still remain anomaly-free (provided the relabelling $d_{R} \leftrightarrow b_{R}$, performed to obtain (6.38) is reversed - a trivial technical point). We now quickly dismiss this model.

\subsubsection{Product Ansatz}

Using (5.22) and (6.38), the mass matrices are easily found to be:

$$
\begin{aligned}
& M_{U} \simeq\left(\begin{array}{ccc}
\epsilon_{1} & \epsilon_{2} \lambda_{f} & \lambda_{f} \\
\epsilon_{1} \lambda_{f} & \epsilon_{2} & \lambda_{f}^{2} \\
\epsilon_{1} \lambda_{f} & \epsilon_{2} \lambda_{f}^{2} & 1
\end{array}\right) \\
& M_{D} \simeq\left(\begin{array}{ccc}
\epsilon_{1} \lambda_{f}^{3} & \epsilon_{2} \lambda_{f}^{4} & \lambda_{f}^{2} \\
\epsilon_{1} \lambda_{f}^{3} & \epsilon_{2} \lambda_{f}^{2} & \lambda_{f}^{4} \\
\epsilon_{1} & \epsilon_{2} \lambda_{f} & \lambda_{f}
\end{array}\right)
\end{aligned}
$$




$$
M_{l} \simeq\left(\begin{array}{ccc}
\epsilon_{1} & \epsilon_{2} \lambda_{f}^{3} & \lambda_{f}^{3} \\
\epsilon_{1} \lambda_{f} & \epsilon_{2} \lambda_{f}^{2} & \lambda_{f}^{4} \\
\epsilon_{1} \lambda_{f} & \epsilon_{2} \lambda_{f}^{4} & \lambda_{f}^{2}
\end{array}\right)
$$

and the stumbling block is immediately eviclent. The $3 \mathrm{rd}$ generation $M_{P}$-scale mass predictions are:

$$
\begin{aligned}
& m_{\tau} \simeq M_{l}(3,3) \simeq \lambda_{f}^{2} \\
& m_{b} \simeq M_{D}(3,3) \simeq \lambda_{f}
\end{aligned}
$$

so that:

$$
\frac{m_{\tau}}{m_{t}} \simeq\left(\frac{m_{b}}{m_{t}}\right)^{2}
$$

This is completely incompatible with the required $M_{P}$-scale order of magnitude prediction:

$$
m_{\tau} \simeq m_{b}
$$

Furthermore, this disasterous prediction remains unchanged in the metric ansatz because the $(3,3)$ elements of the mass matrices are equivalent in all ansätze, being suppressed by only one symmetry ( viz. $\left.U(1)_{f}\right)$. The basic problem is that $U_{1}(1)$ suppressed $M_{D}(1,3)$ whereas $S U_{1}(2)$ does not: this led to the relabelling $d_{R} \leftrightarrow b_{R}$ and explains why $m_{b}$ becomes so high compared to $m_{\tau}$, an insurmountable problem for this model.

\section{Conclusion}

Viewing the Standard Model as the low energy remnant of some extended group $G$, we set out to find which extensions could naturally account for the observed fermion mass and mixing hierarchies. We required that a candidate group $G$ should explain all order of magnitude features of these hierarchies via approximately conserved chiral symmetries: all small numbers in the theory should arise dynamically, none should be entered "by hand". Furthermore, in order to make our search plausible, we demanded that all anomalies should cancel amongst the SM fermions alone. Classifying the possible extended groups 
according to how their irreducible representations were constructed from those of the Standard Model Group, we argued that one particular class of group was favoured: groups whose IRs are identical to those of the SMG. We then found that the non-abelian part of the $S M G$ had to be embedded in the non-abelian part of the extension, $G_{n a}$, as a diagonal subgroup. We argued that $C_{n a}$ consequently had to be a gauge group and further assumed that any abelian components of the extended group were also gauged.

After finding that inter-generation mass gaps were potentially easily accounted for, we were forced to postulate the existence of an entirely new abelian flavour symmetry, $U(1)_{f}$, in order to explain the intra-generation mass gaps. We then examined some subalgebras of the largest candidate group, $S M G^{3} \times U(1)_{f}$, and provided extensive results for 2 particular groups viz. $S U(3) \times S U_{13}(2) \times S U_{2}(2) \times U_{1}(1) \times U_{23}(1) \times U(1)_{f}$ and $S U(3) \times S U(2) \times$ $U_{1}(1) \times U_{2}(1) \times U_{3}(1) \times U(1)_{f}$, with partially conserved symmetries $U_{1}(1) \times S U_{2}(2) \times U(1)_{f}$ and $U_{1}(1) \times U_{2}(1) \times U(1)_{f}$ respectively. The results for the latter were not completely satisfactory, but those for the former successfully achieved our objective of obtaining a natural order of magnitude understanding of fermion masses and mixing angles. It must be noted that while some of the results presented in this paper are good, they nevertheless compare unfavourably with those of the $S M G^{3} \times U(1)_{f}$ model [15], where the PCCSs were $S U_{1}(3) \times U_{1}(1) \times U_{2}(1) \times U_{3}(1) \times U^{\prime}(1)_{f} / U(1)^{\prime}$ and the generator of $U(1)^{\prime}$ is $4 Y_{2}-Y_{3}+Y_{f}$. Further models, obtained by relaxing (6.16) and (6.17), will be presented in a later publication.

\section{Acknowledgement}

HBN acknowledges SERC grant GR/H85496 and EEC grant CS1-D430-C for help with a visit to Glasgow.

GL is grateful for a SERC: studentship. 


\section{References}

[1] S. Glashow, Nucl. Phys. $\underline{22}$ (1961) 571

A. Salam in 'Elementary Particle Theory', ed. N. Svartholm (Almquist and Wiksell, Stockholm, 1968) p367

S. Weinberg, Phys. Rev. Lett. 19 (1967) 1264

[2] J. Pati \& A. Salam, Phys. Rev. D10 (1974) 275

[3] H. Georgi \& S.L. Glashow. Phys. Rev. Lett. 32 (1974) 438

[4] H. Georgi in Proc. of the American Institute of Physics. ed. C.E. Carlson (Meetings at William and Mary College, 1974) p 575

H. Fritzsch \& P. Minkowski. Ann. Phys. $\underline{93}$ (1978) 204

[5] S. Dimopoulos, S. Raby \& F. Wilczek, Phys. Rev. D24 (1981) 1681

S. Dimopoulos \& II. Georgi, Nucl. Phys. B193 (1981) 150

N. Sakai, Z. Phys. C.11 (1981) 153

L.E. Ibanez \& G.G. Ross, Phys. Lett. B10.5 (1981) 439

[6] U. Amaldi, W. de Boer \& H. Furstenau, Phys. Lett. B260 (1991) 447

[7] H. Fritzsch, Phys. Lett. Biㅁ (1977) 436; Phys. Lett. B73 (1978) 317

P. Ramond, R.G. Roberts \& G.G. Ross, 'Stitching the Yukawa Quilt', RAL Preprint RAL93-010 (1993)

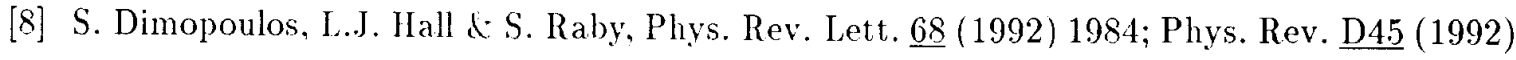
4192

[9] H. Georgi, Nucl. Phys. B156 (1979) 126

[10] A. Davidson, M. Koca \& K.C. Wali, Phys. Rev. Lett. $\underline{43}$ (1979) 92; Phys. Rev. D20 (1979) 421

[11] T. Maehara \& T. Yanagida. Prog. Theor. Phys. $\underline{60}$ (1978) 822

J. Chakrabarti, Phys. Rev. D20 (1979) 2411

F. Wilczek \& A. Zee, Phys. Rev. Lett. $\underline{42}$ (1979) 421 
[12] T. Yanagida in Proc. of the Workshop on Unified Theory and Baryon Number in the Universe, eds. A. Sawada d H. Sugawara (KEK, Tsukuba-Gu, Ibaraki-ken, Japan 1979)

[13] D.S. Shaw \& R.R. Volkas. Phys. Rev. D 47 (1993) 241

[14] C.D. Froggatt, G. Lowe \& H.B. Nielsen, Phys. Lett. B311 (1993) 163

[15] C.D. Froggatt, G. Lowe \& H.B. Nielsen, 'Anti-Grand Unification and the Fermion Mass Problem', Glasgow Preprint GUTPA-93-07-01 (1993), to be published in Nucl. Phys. $\underline{B}$

[16] C.D. Froggatt \& H.B. Nielsen, Nucl. Phys. B147 (1979) 277; Nucl. Phys. B164 (1979) 144

[1T] J. Bijnens \& C. Wetterich. Nucl. Phys. B283 (1987) 237

[18] S. Dimopoulos. Phys. Lett. B129 (1983) 417

G. Anderson et al, 'A Systematic $S O(10)$ Operator Analysis for Fermion Masses', Preprint LBL-33531, UCB-PTH-93/03 (1993)

[19] D.L. Bennett, H.B. Nielsen \& I. Picek, Phys. Lett. B208 (1988) 275

[20] D.L. Bennett \& H.B. Nielsen, 'Fitting the Fine Structure Constants by Critical Couplings and Integers', Proc. of the XXV International Ahrenshoop Symposium on the Theory of Elementary Particles, Gosen, Sept. 1991, NBI Preprint NBI-HE-92-07 (1992)

D.L. Bennett \& H.B. Nielsen, 'Predictions for Nonabelian Fine Structure Constants from Multicriticality', NBI Preprint NBI-IIE-93-22 (1993)

N. Brene \& H.B. Nielsen, Lecture given by HBN at the XVIII Ahrenshoop Symposium on High Energy Physics (Acadamie der Wissenschaften der DDR, Berlin-Zeuthen 1985), NBI Preprint NBI-HE-84-4i (1984)

[21] C.D. Froggatt \& H.B. Nielsen, 'Origin of Symmetries' (World Scientific 1991)

[22] C.D. Froggatt \& H.B. Nielsen, in preparation.

[23] S.E. Rugh \& H.B.Nielsen, 'Why Do We Live In 3+1 Dimensions?', Proc. of the XXVI International Ahrenshoop Symposium on the Theory of Elementary Particles, WendischRietz, Sept. 1992 (Berlin-Zeuthen 1993) DESY 93-013, NBI Preprint NBI-HE-93-11 (1993)

[24] M. Leurer, Y. Nir \& N. Seiberg, Nucl. Phys. B398 (1993) 319 
[25] CDF collaboration. F. Abe et al. Phys. Rev D 155 (1992) 3992

[26] J. Carter, Proc. of the LP-HEP 91 Conference, eds. S. Hegarty, K. Potter \& E. Quercigh (World Scientific 1992) p1

[27] H. Arason $\epsilon t$ al. Phys. Rev. D.46 (1992) 3945

[28] H. Arason et al. Phys. Rev. D4i (1993) 232

[29] Particle Data Group, Phys. Rev. D45 S1 (1992)

[30] L.-L. Chau \& W.-Y. Keung, Phys. Rev. Lett. $\underline{53}$ (1984) 1802 